\title{
TEXTANEIGNUNG IN DER BILDERSPRACHE: ZUM VERHÄLTNIS VON BILD UND TEXT AM BEISPIEL SPÄTMITTELALTERLICHER BUCHILLUSTRATION*
}

\author{
VON LIESELOTTE E. SAURMA-JELTSCH
}

\section{Die Sonderstellung der Illustration}

In der Buchillustration ${ }^{1}$ ist das Verhältnis zwischen Bild und geschriebenem Text ein ganz besonderes. Ihrer Begrifflichkeit nach dient die Illustration der Auszeichnung, Erleuchtung, Inspiration wie auch der Erläuterung ${ }^{2}$ eines Textes, der veranschaulicht werden soll. Sie steht damit in einem viel stärkeren Maße als andere Kunstwerke im Zusammenhang mit Text. Ihre Funktion wird denn üblicherweise auch als Dienst an diesem bezeichnet, woraus - etwa im Katalog einer Ausstellung zur Trivialillustration in Cambridge 1986 - eine Äquivalenz, ja eine Austauschbarkeit von Bild und Text abgeleitet wird: „It will be the expression in drawn-lines of something that might otherwise be expressed in words" "3. Auf die Problematik eines solchen Funktionsverständnisses soll zunächst an einem allgemein vertrauten Muster von Bild und Text eingegangen werden, bevor einzelne Arbeitshypothesen erarbeitet werden.

Wie wenig die These einer Äquivalenz die Sonderstellung der Illustration umschreibt, mag die Analyse der drei folgenden Druckversionen des Suppen-Kaspars von Heinrich Hoffmann belegen ${ }^{4}$ : Die wegen ihrer Aufnahme in die besonders gefeierte, 100. Auflage des Werkes im Jahre 1876 (Abb. 1) lange gültige Version ${ }^{5}$ sei mit der jüngsten Fassung konfrontiert (Abb. 3).

* Bei diesem Text handelt es sich um eine überarbeitete Fassung eines in Basel im Wintersemester 1987/88 im Rahmen einer Ringvorlesung zum Thema „Bild und Wort" gehaltenen Vortrags. Für Anregungen im Zusammenhang einer ersten redaktionellen Durchsicht sei Herrn Prof. Dr. G. Schmidt, Wien, sehr gedankt.

1) Da im folgenden eine Funktions- und keine Gattungsanalyse vorgenommen wird, ist mit „Buchillustration" der umfassendere Begriff für eine „bildliche ... Darstellung, die die anschauliche Ergänzung eines Worttextes“ (J. JAHN, Wörterbuch der Kunst, Stuttgart 1966, S. 314) meint, gewählt worden, obwohl dic hier behandelten Formen spätmittelalterlicher Buchausstattung nach streng kunsthistorischer Terminologie als Buchilluminationen bezeichnet werden müßten. Zu dieser Begriffsverwendung s. H. Frohmorghen-Voss, Text und Illustration im Mittelalter (= Münchner Texte und Untersuchungen zur dt. Lit. des Mittelalters Bd. 50), München 1975.

2) Zum Wortfeld „illustratio“ s. etwa J. F. NierMeYer, Mediae Latinitatis Lexicon Minus, Leiden 1976, S. 510.

3) Sing a Song for Sixpence, Ausst. British Library London 1986/87, S. 13. - Unter den unzähligen Belegen für dieses Verständnis sei nur der differenzierende Ansatz von Wilhelm Messerer erwähnt, welcher der Illustration die Möglichkeit einräumt, „Ubersetzung in eine andere Sprache zu sein“ und ,als Sprache im Vollsinne ein Äquivalent zum Text“ darzustellen. W. Messertr, Illustrationen zu Wernhers „drei Liedern von der Magd“, in: Chr. Cormeau (Hrsg.), Deutsche Literatur im Mittelalter, Kontakte und Perspektiven, Stuttgart 1979, S. 447-472, bes. S. 447.

4) Es scheint bisher noch keine systematische Untersuchung der verschiedenen Versionen vorgenommen worden zu sein. Eine orientierende Übersicht über einzelne Ausgaben bei H. DALBERT, Lexikon der Kinder- und Jugendliteratur, Bd. 3, Basel/ Weinheim 1979, S. 483-488, dort weitere Literatur; K. DoDtRt:R und H. MUl.t.R, Das Bilderbuch, Weinheim/ Basel 1973, bes. S. 152 ff.; J. JAHN, Kommentar zum Neudruck der 24. Auf., Frankfurt 1979, bes. S. 59 ff.; M.-L. KonNecker, Dr. Heinrich Hoffmanns „Struwwelpeter“. Untersuchungen zur Entstehungs- und Funktionsgeschichte eines bürgerlichen Bilderbuchs, Stuttgart 1977, S. $122 \mathrm{ff}$.

5) Die hier vorgestellte Version des Suppen-Kaspar in der 100. Ausgabe konnte - allerdings anhand einer nicht 
Als Orientierungspunkt dient die vom Autor selber akzeptierte Fasson (Abb. 2) von 1847, in der noch unveränderten 24. Auflage ${ }^{6}$. Daß eine besonders enge Relation zwischen Bild und Text beabsichtigt ist, läßt uns die Übereinstimmung der Leserichtung in den beiden Medien vermuten. Jeweils ein Bild entspricht einem Textabschnitt, der Ablauf der Ereignisse wird im Bild, dem Text entsprechend, von links nach rechts bzw. von oben nach unten abgelesen. In den zwei älteren Fassungen wird diese unmittelbare Zusammengehörigkeit durch eine Numerierung der Bilder betont; in der ,Urfassung sogar durch eine optische Zäsur in Schrift und Bild zwischen dem ersten, ursächlichen Erzählabschnitt und den daraus sich ergebenden weiteren. Zumindest in den zwei jüngeren Exemplaren haben wir es mit intendierten ,Ikonen' zu tun, die Bild wie Text in der seit Generationen vertrauten Weise wiedergeben wollen. Dennoch sind Unterschiede zu verzeichnen, die uns in den hier vorgestellten Ausgaben Bild und Schrift als jeweils unverwechselbare Einheit erkennen lassen. Dies fängt bei der Gestaltung der Seite an, die in der 100. Auflage gegenüber dem Original ein Bild- und Schriftkontinuum herstellt und damit auf die Formel der akzentuierten Einführung verzichtet. Das hier die Bilder umrahmende Rankenwerk führt den Betrachter ohne Unterbruch zum Endpunkt der Erzählung, zu den Blumen auf dem Grabe Kaspars. Die Version der jüngsten Ausgabe, die, wie das Titelblatt verspricht, „nach der Urfassung neu gezeichnet" worden sein soll, hat zwar die originale Anordnung einer Isolierung des ersten Bildes und einer zusammenfassenden Begleitung der übrigen Szenen durch kalligraphische Linien übernommen, verliert aber durch den Verzicht auf die optische Absetzung der Einleitungsszene das dramatische Element der Exposition. Verändert hat sich vor allem die Intensität der erzählerisch-symbolischen Darstellungsmittel. Diese sind wohl am eindrücklichsten in der 100. Auflage gestaltet, die dem Betrachter die schrecklichen Folgen des Ungehorsams besonders drastisch vor Augen führen will. Mit geradezu hypnotischer Zwangsläufigkeit entwickelt sich hier aus dem gesundheitsstrotzenden der immer schwächer protestierende, schließlich vom Grab gleichsam angezogene Kaspar. Stilistische wie auch ikonographische Veränderungen dramatisieren die Erzählung und unterstützen ihren appellativen Charakter. So ist das Grab, das im Original nur symbolisch angedeutet wird, hier ein eigener szenischer Abschnitt geworden, ist es doch eigens mit Blumen geschmückt und steht auf ihm nicht bloß das Kreuz mit dem Namenszug des Verblichenen, sondern auch noch das Corpus delicti, die Suppenschüssel. Fin solcher Appell fehlt in der originalen Fassung, ihre Darstellungen sind durch größere Zeichenhaftigkeit und knappe Farbigkeit eigentliche Schriftbilder und sprechen damit vor allem die Memorierfähigkeit des Betrachters an ${ }^{7}$. Auch die jüngste Ausgabe verzichtet weitgehend auf eine Steigerung des Grauens, friert allerdings die ,realistischen“ Elemente der Urfassung wie Gesichtsausdruck, Haarbildung und Figurenhaltung noch stärker ein und gestaltet durch eine Rhythmisierung von Formen und Farben die Seite als dekoratives Ganzes.

ganz vollständigen Sammlung - erstmals 1865 beobachtet werden. Es scheint, daß die tiefsten Eingriffe 1858 vorgenommen worden sind. Allerdings können sich die Veränderungen nicht, wie stereotyp zu lesen ist, allein aus der Umstellung von der Lithographie auf den Holzschnitt exklären lassen, handelt es sich doch deutlich um gravierende inhaltliche Veränderungen. JAHN, Kommentar (zit. Anm. 4), macht denn zu recht darauf aufmerksam, daß die Idealisierung den ursprünglichen Intentionen Hoffmanns gänzlich zuwiderläuft.

6) Die uns vertraute Zusammenstellung der Geschichten ist erst in der 5. Auflage (1847) gefunden worden. Diese wiederum ist Grundlage für die 24., die immer noch unter der Kontrolle Hoffmanns entstanden und nach seinen Bedingungen koloriert worden ist; s. JAHN, Kommentar (zit. Anm. 4), S. 64.

7) Zur mnemotechnischen Funktion der Darstellungen s. vor allem DoDERter und MOL.L.t, Bilderbuch (zit. Anm. 4), S. $156 \mathrm{ff}$. - Hoffmann selber weist in seinem Vorwort zur 100. Ausgabe auf die Bedeutung der leichten Erlernbarkeit sowohl seiner Verse als auch Bilder hin. 
Gerade wegen des gewünschten Zitatcharakters der Darstellungen sind die Änderungen beinahe unauffällig, und dennoch sind sie Ausdruck eines tiefgreifenden Wandels in der Ansprache $^{8}$ an den Benützer. Ohne die Interpretation allein von der Analyse dieser drei Ausgaben überstrapazieren zu wollen ${ }^{9}$, scheinen sich doch in ihnen jeweils grundsätzlich verschiedene Aussagerichtungen unterscheiden zu lassen.

Als Motivation für die Schaffung seines Werkes gibt Hoffmann ${ }^{10}$ an, er habe sich über die moralischen Vorschriften und Räsonnements geärgert, welche die damalige Kinderliteratur geprägt hätten, deren Bilder überdies allzu trefflich und glänzend bemalt seien. Mit seinem Werk verfolgt er denn vor allem didaktische Zwecke, die - wie Jahn ${ }^{11}$ belegen kann - mit einer von Jean Paul propagierten Pädagogik zusammenhängen dürften, wonach der Vorstellungskraft und Phantasie des Kindes mehr Raum gegeben werden sollte. Mit seinem Grundkonzept allerdings - auf didaktischem Weg moralische Inhalte zu konkretisieren - fügt sich Hoffmanns erste Fassung des Werkes (Abb.2) nahtlos in die Kinderliteratur der Aufklärung ${ }^{12}$ : Es ist ein Belehrungsbuch, in dem das Bild der leichteren Einprägsamkeit des Textes dienen soll. In einem ganz anderen Funktionszusammenhang scheint die 100. Ausgabe zu stehen: Hier sollen die moralischen Inhalte drastisch vor Augen geführt und eindrucksvoll nachvollziehbar gemacht werden: Das Bild ist damit in den Sog der in der Kinderliteratur schon etwas früher zu beobachtenden Tendenz zu einer Intimisierung geraten. Richter ${ }^{13}$ vermag diesen Prozeß in Zusammenhang zu bringen mit einer Veränderung sowohl der Familienstruktur als auch der Rezeptionsweisen. Während nämlich im 18. Jahrhundert die primär didaktischen Inhalte für einen Dialog zwischen Autoritäten und Kind bestimmt waren, werden im Lauf des 19. Jahrhunderts die Bücher zunehmend privater und emotionalisierter und richten sich nun an einen neuen Adressaten, an das Kind. Spiegelt der Unterschied zwischen den beiden älteren Versionen vor allem die veränderte Gebrauchssituation, so dürfte sich in den jüngeren Ausgaben (Abb. 3) überdies ein Normenwandel ausdrücken. Wohlgenährtheit als Erziehungsmaxime ist jedenfalls im 19. Jahrhundert ein nicht hinterfragter Wert ${ }^{14}$, ebenso wenig wird in der Erziehung an Durchsetzungsformen, welche dem Unfolgsamen mit Schrecknissen drohen, gezweifelt ${ }^{15}$. Beide

$\left.{ }^{8}\right)$ Der Begriff der Ansprache eines Bildes oder einer Illustration geht von der Prämisse aus, das Kunstwerk gehöre zu den Kommunikationsmitteln und richte sich mit der ihm eigenen „Sprache“ (Bildsyntax, Stilmittel etc.) an den Betrachter mit einem ganz bestimmten, vorgegebenen Leseverständnis. - Zu dieser Begriffsverwendung s. H. BEI.TING, Wandmalerei und Literatur im Zeitalter Dantes, in: Poetik und Hemeneutik, 12, 1987, S. 53-79, bes. S. 60 f.; zum Verhältnis Sprache-Kunstwerk-Rezipient s. W. KEMP, Sermo Corporeus. Die Erzählung der mittelalterlichen Glasfenster, München 1987, Teil II; zum Verständnis des Kunstwerks als Kommunikationsmittel s. ebda. Teil IIl und Ders., Der Anteil des Betrachters. Rezeptions-ästhetische Studien zur Malerei des 19. Jahrhunderts, München 1983, bes. S. $29 \mathrm{ff}$.

9) Mit Ausnahme der als solche auch gekennzeichneten Neuschöpfungen existieren mindestens fünf, deutlich voneinander abweichende Versionen, die sich alle auf die originale Fassung berufen. - Für die Hilfe bei der Sichtung dieses Materials sei dem Schweizerischen Jugendbucharchiv in Zürich und vor allem Frau Anna Katharina Ulrich sehr herzlich gedankt.

10) H. Hoffmann, Wie der „Struwwelpeter“ entstand, in: Gartenlaube, 1871, Nr. 46.

11) JAHN, Kommentar (zit. Anm. 4), S. 56.

12) D. Richter, Das fremde Kind. Zur Entstehung der Kindheitsbilder des bürgerlichen Zeitalters, Frankfurt 1987, S. 107; R. Wı.D, Die Vernunft der Väter. Zur Psychographie von Bürgerlichkeit und Aufklärung in Deutschland, Stuttgart 1987 , S. $47 \mathrm{ff}$.

13) Richter, ebda. S. $101 \mathrm{ff}$.

14) Zu den Zusammenhängen von Kindersterblichkeit als Bedrohung der familiären Existenz s. Wur.D, Vernunft der Väter (zit. Anm. 12), S. $156 \mathrm{f}$. bzw. zur Ausgrenzung der Armut ebda. S. 73 ff.

15) Zur Gehorsamkeitspflicht s. ebda., S. 281 ff; die Strukturen repressiver Erziehung am Beispiel des Struwwelpeters erörtert die Arbeit von KOnNECKER, Struwwelpeter (zit. Anm. 4). 
Prinzipien haben in unserer Zeit eine völlige Neueinschätzung erlebt und gehören für den heutigen Betrachter wohl nicht mehr in den Bereich seiner Alltagserfahrung. Das Bild wird hier zum Bildungs- und Unterhaltungsgegenstand für das Kind, dem - wie der Rückgriff auf die biedermeierlichen Kostüme belegt - Geschichten aus einer fremden Welt vermittelt werden sollen.

Diese drei Beispiele eines vermeintlich über lange Zeit und in einer unermeßlich großen Anzahl von Ausgaben übermittelten unveränderten Bild-Text-Verhältnisses haben gezeigt, daß von einer Austauschbarkeit der beiden Medien nicht die Rede sein kann. Während der Text keinerlei Eingriffe erlebt, reagiert das Bild deutlich auf den Mentalitätswandel. Es vermittelt hier eine konkrete Ansprache an einen ganz bestimmten Betrachter oder Betrachterkreis, während der Text über eine ganz andere Multifunktionalität verfügt.

\section{Hypothesen zum Verbältnis der beiden Medien}

Der solcherart gewonnene Vorbehalt gegenüber der These einer Äquivalenz von Text und Illustration soll zusammen mit drei weiteren Hypothesen an der Gattung der spätmittelalterlichen Buchmalerei überprüft werden. Diese lauten: 1. Das Bild steht nicht einseitig in einem Dienstleistungsverhältnis zum Text, sondern bildet mit diesem gemeinsam Teil eines Diskurses zwischen Gruppen oder Individuen und der Schriftlichkeit insgesamt. Beide Elemente gehören in den größeren Bereich der Kommunikation und reflektieren deren zeit-, gruppen- und individualspezifischen Wandel. Ein Beispiel für diese Relation hat bereits der Suppen-Kaspar geliefert. Was die Bedeutung der Schriftlichkeit betrifft, so läßt sich der Wandel etwa an den Comics erkennen, in denen sich eine Verschiebung zugunsten des Bildes und des in der Sprechblase verschriftlichten gesprochenen Wortes ereignet hat.

Dieser ersten Hypothese soll die zweite Behauptung folgen: 2. Das Bild ist anpassungsfähiger als der Text, insbesondere wenn es sich um tradierten Text handelt, und hat unmittelbarer die Möglichkeit einer direkten Ansprache an die Rezipienten. Das Bild vermag also den Text für bestimmte Benützer neu aufzubereiten, ihrem Verständnis näher zu bringen. Der Ausgangspunkt dieser zweiten Behauptung ist im folgenden an einigen Beispielen genauer zu erörtern.

Dieselbe Textstelle aus dem Trojanerkrieg Konrads von Würzburg ${ }^{16}$, das Fest der Pallas Athene, an dem die Begegnung zwischen Achilles und Deidamie stattfindet, ist in den beiden ungefähr gleichzeitig entstandenen Versionen in Berlin (Abb.4) und Nürnberg (Abb. 5) illustriert $^{17}$. Trotz gewisser äußerer Übereinstimmungen - in der Berliner Handschrift scheint etwa das Architekturmotiv der Nürnberger Darstellung in verkürzter Form wiedergegeben - sind

${ }^{16}$ ) V. 14537 ff. A. v. KEJ.l.er, Der Trojanische Krieg von Konrad von Würzburg (= Bibliothek des litrerarischen Vereins, Bd. 44), Stuttgart 1858.

$\left.{ }^{17}\right)$ Berlin, Staatsbibliothek, Preußischer Kulturbesitz, ms. germ. fol. 1. Nach den Wasserzeichen dürfte die Handschrift in die Zeit von 1443-1446 zu datieren sein, sie wurde in der Werkstatt Diebold Laubers von Hagenau hergestellt; s. H. Wegener, Beschreibendes Verzeichnis der Miniaturen und des Initialschmucks in den deutschen Handschriften bis 1500 ( = Beschreibende Verzeichnisse der Miniaturen-Handschriften der PreuBischen Staatsbibliothek zu Berlin), Leipzig 1928, S. 32-35; Ausst.-Kat. Zimelien. Abendländische Handschriften des Mittelalters aus den Sammlungen der Stiftung Preußischer Kulturbesitz Berlin. Ausst. Berlin 1975 - 1976, Wiesbaden 1975, S. 142, dort weitere Literatur. - Nürnberg, Germanisches Nationalmuseum, Hs. 998. In der Handschrift befindet sich die Daticrung 1441; s. L. KURRAs, Die deutschen mittelalterlichen Handschriften. Teil 1 ( = Katalog des Germanischen Nationalmuseums Nümberg. Die Handschriften des germanischen Nationalmuseums Nürnberg Bd. 1,1), Wiesbaden 1974, S. $9 \mathrm{f}$. 
die Inhalte auf den ersten Blick als zwei ganz verschiedene erkennbar. Während in Berlin Achilles und Thetis die mit Blütenzweigen beschäftigte Deidamie und ihre Gefährtin im Tempel beobachten $^{18}$, ist die Nürnberger Version wesentlich komplexer strukturiert. Auch dem mit den Bildgewohnheiten des 15. Jahrhunderts unvertrauten Betrachter eröffnet diese Darstellung Assoziationen etwa an einen Götzendienst oder einen Gesellschaftstanz. Das Ereignis der Begegnung wird peripher behandelt und erscheint gleichsam eingebettet in ein dichtes Netz bildnerischer Interpretationen. Hier wie dort ließe sich die Botschaft der Bilder nur mit sorgfältigen Analysen der Bildersprache dieser Codices eruieren ${ }^{19}$. $\mathrm{Da}$ aber in beiden Darstellungen jeweils zwei ganz verschiedene Vorgänge gewählt werden, um dem Leser das Thema der Begegnung von Achilles und Deidamie zum Fest der Athene zu illustrieren, darüber kann kein Zweifel bestehen.

Noch deutlicher wird dies bei einem Bildvergleich einer verwandten Thematik aus den Lauberhandschriften mit einem zwar zeitgenössischen, aber ganz anders orientierten Werk. Das Fest der Venus als Stätte der geschichtswirksamen Begegnung zwischen Paris und Helena wird in der Würzburger Trojanerkrieg-Handschrift aus den 50 er Jahren ${ }^{20}$ als kühne Schiffahrt des Helden zu der vor den Tempeltoren wartenden Helena gestaltet (Abb. 6). An der wohl zeitgenössischen Version (Abb. 7) des Martinus Opifex in der Wiener Handschrift ${ }^{21}$ läßt sich nicht allein die Andersartigkeit der Situationsbenennung erkennen, sondern in diesem Festzug wird auch mit einer ganz anderen Sprache gearbeitet. Von den hier vorgetragenen kompliziert verschlüsselten Anspielungen soll einzig auf das scheuende Pferd am rechten unteren Bildrand aufmerksam gemacht werden, auf dem ein mit einem Minnekranz geschmückter Narr, auf dessen Schulter ein Affe hockt, aus dem Bild herauszustolpern scheint. Es dürfte hier die irdische Leidenschaft und Torheit gemeint $\operatorname{sein}^{22}$, deren zwieträchtiges Wirken in ihrer, dem Bildzentrum entgegengesetzten Position zum Ausdruck kommt. Das hier angekündigte Unheil zieht geradezu den Betrachter in die Geschehnisse hinein, scheinen deren Akteure doch auf ihn zuzukommen.

An diesem Beispielpaar wird denn auch deutlich, daß nicht allein die verschiedenen Inhalte, sondern vor allem deren Ausgestaltung oder besser Anordnung im Bildganzen entscheidend

\footnotetext{
18) Zum Motiv des Blütenzweigsammelns als Ausdruck gottgefalliger und liebender Betätigung s. die kuriose Umdeutung des Bekehrungserlebnisses Parzivals bei Trevrizent in einer zeitgleichen, ebenfalls aus der Lauber-Werkstatt stammenden Parzivalhandschrift: Wien, Österreichische Nationalbibliothek, Cod. 2914, fol. 315v, Abb. s. B. Scrirok, Wolfram von Eschenbach „Parzifal“. Die Bilder der illustrierten Handschriften (= Litterae. Göppinger Beiträge zur Textgeschichte, hrsg. von U. Muluter u. a., Nr. 67) Göppingen 1985, S. 30.

19) $\mathrm{Zu}$ den Besonderheiten der Erzählweise in der Lauberwerkstatt s. am Beispiel der Parzival-Handschriften L. E. STAMM-SAURMA, ,Zuht und wicze": Zum Bildgehalt spätmittelalterlicher Epenhandschriften, in: Zeitschr. des deutschen Vereins für Kunstwiss., XLI, 1987, S. 42-70. - Weitere Modi der Erzählung sollen in der Úbersichtsarbeit zu den Werkstätten von 1418 und Diebold Lauber dargestellt werden.

20) Würzburg, Universitätsbibliothek, M. ch. f. 24, fol. 176v; s. H. THURN, Die Handschriften der Universitätsbibliothek Würzburg, Bd. 2: Die Handschriften aus benediktinischen Provenienzen 1, Wiesbaden 1973, S. 50-53. - Zu den V. 19389 ff. kennt die Berliner Handschrift (vgl. Anm. 17) eine verwandte, allerdings chiffrenhaftere Darstellung auf fol. 184; in beiden Codices folgt anschließend die Begegnung zwischen Paris und Helena.

${ }^{21)}$ Wien, Österreichische Nationalbibliothek, Cod. 2773. Die Handschrift muß vor 1456 entstanden sein; s. Regensburger Buchmalerei. Ausst. Regensburg 1987, Kat. Nr. 100, S. 109, dort weitere Literatur. - Guddo Da CortunA, Historia Destructionis Troiae, lib. 7; Ed. N. E. Griffin, Cambridge Mass. 1936, S. 70.

22) Zur Bedeutung des Affen als Symbol der Sexualität s. H. W. JANson, Apes and Ape Lore in the Middle Ages and the Renaissance ( = Studies of the Warburg Institute, Vol. 20) London 1952, S. 261 ff.; der Narr als Verkörperung von Lasterhaftigkeit und Dummheit s. Lexikon der christlichen Ikonographie, hrsg. von E. Kirschbacm u. a., 3, Rom/ Freiburg/Basel/Wien 1971, Sp. 314-318.
} 
die Art der Ansprache einer Illustration definieren. Die Wahl der Mittel, Federzeichnung oder Deckfarbenmalerei mit Hintergrund und Rahmen, wie auch die Anordnung der Formen im Bildfeld und im Verhältnis zur Schrift ergeben eine dem Bild eigene Syntax. In ihr kommt am ehesten das Anspruchsniveau der Handschrift zum Ausdruck ${ }^{23}$. Daß es hier um weit mehr geht als um den Unterschied von prunkvoller Ausführung und einfacher Gestaltung, oder auch von künstlerisch hochstehender oder mediokrer Ausführung, lassen die verschiedenen Inhalte sowie deren jeweils unterschiedliche Bildsyntax in den Illustrationen zum Fest der Venus vermuten.

Als dritte Hypothese soll denn hinzugefügt werden: 3. Die Bildsyntax sowie das Bildprogramm bereiten den Text für ganz bestimmte Verstehensweisen auf, wobei die Effektivität von der Qualitätsfrage unabhängig ist. Diese dritte Annahme würde bedeuten, daß etwa Bilderrätsel oder Comics genauso effizient Informationen zu vermitteln vermögen wie beispielsweise die verschlüsselte Miniatur zum Fest der Venus in der Wiener Handschrift.

Die drei Hypothesen seien hier nochmals benannt: 1. Illustration und Text bilden ein gemeinsames Ganzes, das den Wandel der Beziehung zur Schriftlichkeit bzw. zum Bildverständnis reflektiert; 2. die Bilder sind anpassungsfähiger und vermögen unmittelbarer neue Verständnisschichten zu präsentieren; 3. Bildsyntax und Programm bieten für einen bestimmten Rezipienten oder eine Rezipientengruppe ein spezifisches Interpretationsmuster an, dessen Aussagekraft weitgehend unabhängig von der Qualität der Darstellungen ist.

\section{Schrift und Bild im mittelalterlichen Verständnis}

Bevor diese Thesen weiter überprüft werden, müssen einige allgemeine Grundlagen zum Verhältnis von Schrift und Bild im mittelalterlichen Wertsystem in Erinnerung gerufen werden, um vor diesem Hintergrund den Übergang zu den neuen volkssprachlichen Beispielen klarer darzustellen zu können.

Die wohl bekannteste Aussage hierzu ist zweifellos diejenige Gregors des Großen, in welcher die Bilder als die Bücher der, illiterati‘ bezeichnet werden; ein Diktum, das bis ins 15. Jahrhundert in unzähligen Varianten vorgetragen wird ${ }^{24}$. Bis zu diesem Zeitpunkt bleibt im wesentlichen die Priorität der Schrift als des übergeordneten Erkenntniswegs gültige Lehrmeinung. Allerdings

${ }^{23}$ ) Zum Verhältnis Ausstattung und Anspruchsniveau s. N. H. OTT, Typen der Weltchronik-Ikonographic. Bemerkungen zu Hllustration, Anspruch und Gebrauchssituation volkssprachlicher Chronistik aus überlieferungsgeschichtlicher Sicht, in: Jb. der Oswald von Wolkenstein Gesellsch., hrsg. von H.-D. MÜcK und U. MU...ER, 1, 1980/81, S. 29-55, bes. S. 30-34; Ders., Úberlicferung, Ikonographie - Anspruchsniveau, Gebrauchssituation. Methodisches zum Problem der Beziehung zwischen Stoffen, Texten und Illustrationen in Handschriften des Spätmittelalters, in: Literatur und Laienbildung im Spätmittelalter und in der Reformationszeit, Symposion Wolfenbüttel 1981, hrsg. von L. Grenzmann und K. Stackmann, Stuttgart 1984, S. $356-386$, bes. S. $363 \mathrm{ff}$.

${ }^{24}$ ) Im Brief an Serenus von Marseille, s. Monumenta Germaniae Historica Epistolarum t. II, Berlin 1899, S. 195 (ep. IX 208): Idcirco enim pictura in eclesiis adbibetur, ut bi qui litteras nesciunt saltem in parietibus videndo legant, quae legere in codicibus non valent. A hnlich im zweiten Brief an Serenus (ep. XI 10), s. ebda. S. 270: Nam quod legentibus scriptura, boc idiotis praestat pictura cermentibus, quia in ipsa ignorantes vident, quod sequi debeant, in ipsa legunt qui litteras nesciunt; unde praecipue gentibus pro Lectione pictura est. Dazu s. W. Durig, Imago. Ein Beitrag zur Terminologie und Theologie der Römischen Liturgie (= Münchner Theol. Studien. Il. Systemat. Abt., Bd. 5), München 1952, S. 51 f.; zur Weitertradierung dieses Diktums Gregors d. Gr. s. die Zitatensammlung von L. Gougaud, Muta praedicatio, in: Revue Bénédictine, 1930, 42, S. 168 - 171; s. auch M. CAMLL.E, Seeing and Reading: Some Visual Implications of Medieval Literacy and Illiteracy, in: Art History, 8,1985, S. $26-49$, bes. S. 26 und $32 \mathrm{ff}$. 
wird den Bildern allmählich ein breiteres Spektrum an Funktionen zuerkannt als bloße Unterrichtsmittel zu sein. Thomas von Aquin etwa beschreibt ihren Sinn folgendermaßen: „Ein dreifacher Grund veranlaßte die Einführung der Bilder in der Kirche. Zunächst die Unterweisung der Ungebildeten, für welche die Bilder gleichsam belehrende Bücher sind. Der zweite Grund war der, daß das Mysterium der Menschwerdung und das Beispiel der Heiligen dem Gedächtnis lebendiger nahe gebracht werden. Der dritte, daß die Frömmigkeit angeregt werde, die durch das Geschaute stärker beeinflußt wird als durch das Gehörte. " 25 Bilder dienen somit nicht nur der Belehrung der Unwissenden, sondern auch der Förderung der Andacht und sind Erinnerung an das Beispiel der Heiligen. Betont wird hier insbesondere die stärkere Eindringlichkeit, die dem Bild gegenüber dem Wort - dem Auge gegenüber dem Ohr - zukommt. Noch stärker vertritt diese Position Durand de Mende, der ausdrücklich auf die Unmittelbarkeit des Mediums aufmerksam macht, das direkt über das Auge auf die Seele wirke und nicht über die Vermittlung des Ohres ${ }^{26}$.

Der Exempelwert und die - auch emotionale - Vertiefung der Andacht sollten in der Folgezeit den Stellenwert des Bildes in den Lehrmeinungen, vor allem aber in seiner Verwendung entscheidend verändern. Außerdem wird ein Vorstellungsbereich wirksam, der bereits bei den Vätern, so etwa im Imago-Begriff des Ambrosius, eine große Rolle gespielt hat: die Teilhabe. Danach ist das Bild nicht bloß eine funktionale Darstellung einer Sache, sondern auch ,eine Ausstrahlung, ein Sichtbar- und Offenbarwerden des Wesens mit substantieller Teilhabe ... am Gegenstand. Bild ist nicht als wirklichkeitsfremde, nur im Bewußtsein vorhandene Größe zu

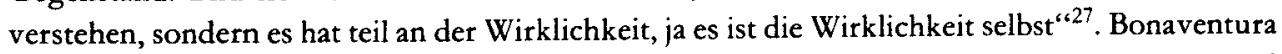
etwa zählt die Bilder unter die signa divinitus data ..., unter die göttlich gegebenen Zeichen, und fährt weiter, sie seien als abgebildete Beispiele dem noch rohen und im Sinnlichen befangenen Geist vorgelegt, damit sie über das Sinnliche zum Geistigen getragen würden, tamquam per signa ad signata, gleichsam vom Zeichen zum Bezeichneten ${ }^{28}$. Diese dem mittelalterlichen Denken durchaus geläufige Trennung von res und signa, wie sie üblicherweise auf das Wort angewendet wird $^{29}$, hat hier dem Bild die Qualität zugesprochen, an dem übergeordneten Größeren, dem Bezeichneten, als Zeichen teilhaftig zu sein, ein Zusammenhang, der vor allem für die Mystik eine große Rolle spielen wird.

In den Lehrmeinungen allerdings ändert sich die Definition des Verhältnisses zwischen Schrift

25) Ubersetzung nach DURig, ebda., S. 51. Th. v. Aquin, Kommentar zu den Sentenzen des Petrus Lombardus (III Sent. d IX q. 1 a. 2 sol. 2 ad 3): Fuit autem triplex ratio institutionis imaginum in Ecclesia: Primo ad instructionem rudium, qui eis quasi quibusdam libris edocentur. Secundo ut incarnationis mysterium et sanctorum exempla magis in memoria essent, dum quotidie oculis repraesentantur. Tertio ad excitandum devotionis affectum, qui es visis efficacius incitatur quam ex auditis.

26) Durandus von Mende, Rationale, lib. 1, cap. III, I, 4: Picturae et ornamenta in Ecclesia sunt laicorum lectiones et scripturae ... Pictura namque plus videtur movere animum quam scriptura. Per picturam quidem res gesta ante oculos ponitur; sed per scripturam res gesta quasi per auditum qui minus movet animum ad memoriam revocatur; zitiert nach GOUGAUD, Muta praedicatio (zit. Anm. 24), S. 169.

27) Dorig, Imago (zit. Anm. 24), S. 60 f, dort auch weitere Entwicklung des Vorstellungsbereiches Imago als Bildwirklichkeitsaussage.

${ }^{28}$ ) Itinerarium mentis ad deum II, 11: Signa divinitus data ... (vel picturae; simulacra et spectacula) ... sunt exemplaria vel potius exemplata, proposita mentibus adbuc rudibus et sensibilibus, ut per sensibilia, quae vident, transferantur ad intelligibilia, quae non vident, tamquam per signa ad signata; zitiert nach R. SUCkAl.t, Arma Christi. Überlegungen zur Zeichenhaftigkeit mittelalterlicher Andachtsbilder, in: Städel-Jb., 6, 1977, S. 177-208, bes. S. 188.

$\left.{ }^{29}\right)$ Zur Ubertragbarkeit dieses Teilhaftigkeitsbegriffs vom Wort auf das Bild s. CAmIJ..., Seeing and Reading (zit. Anm. 24), bes. S. $30 \mathrm{ff}$. 
und Bild nur unwesentlich. Auch für Bonaventura dient ja die emotionale Teilnahme, welche durch das Bild gefördert wird, lediglich als Einstieg zur eigentlichen Erkenntnis, die über den Geist führt. Dasselbe Verständnis des Bildes vertreten noch Theologen im 15. Jahrhundert, wie etwa Johannes Gerson ${ }^{30}$. Gert de Groote allerdings trifft bereits eine Einschränkung, insofern er den Bildergebrauch lediglich für den privaten Bereich ablehnt, wogegen er ihn im öffentlichen durchaus tolerieren mag. Damit trägt er der gegenüber dem 13. Jahrhundert veränderten Praxis im Umgang mit Bildern Rechnung ${ }^{31}$. Wie hinlänglich bekannt ist, waren an diesem Wandel ganz wesentlich die Mystiker beteiligt, deren an Bildern reiche Sprache den Unterschied zwischen Bild- und Sprachbetrachtung verschliff ${ }^{32}$. Die zunehmende Austauschbarkeit von Bild und bildlicher Vision wird durch eine Reihe von Geschichten belegt, in denen jeweils von einem lebendig gewordenen Bild die Rede ist ${ }^{33}$. Die Begegnung mit dem Heiligen ist als bildliche Vorstellung real geworden, was zugleich eine Individualisierung des Bilderlebnisses bedeutet: Das Bild als Meditationsgrundlage, teilhaftig des übergeordneten Bezeichneten, hat damit nicht nur eine Bedeutungserhöhung erlebt, sondern auch den Verwendungswandel zum privaten Bild durchgemacht ${ }^{34}$.

In unserem Zusammenhang muß die Veränderung der Gebrauchsart von Texten ebenfalls kurz gestreift werden. Die Zunahme der Schriftlichkeit, die sich im Laufe des 11. und 12. Jahrhunderts beobachten läßt ${ }^{35}$, basiert auf einer Anerkennung des geschriebenen Wortes als Äquivalent zu dem bis dahin primär gültigen ges prochenen Wort, so etwa im Rechtswesen. Diese Entwicklung verleiht dem Medium ein neues Gewicht. Das private Lesen allerdings, das, wie wir aus seiner häufigen Erwähnung als literarischem wie auch als bildlichem Topos wissen ${ }^{36}$, nicht selten anhand liturgischer Schriften erlernt wird, erlebt den für unseren Zusammenhang entscheidenden Wandel im Zuge der zunehmenden Volkssprachlichkeit des 13. Jahrhunderts ${ }^{37}$. Es entsteht nun eine Gruppe von ,illiterati', also des Lateinischen Unkundigen, die gleichwohl über eine beträchtliche Bildung verfügen und durchaus die volkssprachliche Schrift beherrschen, nicht selten zumindest über minimale Lateinkenntnisse verfügen. Wie intensiv die Kirche darauf reagiert hat, verrät der Häresievorwurf, den der Bischof von Sens 1210 gegen jegliche Verwendung volkssprachlicher theologischer Literatur erhebt ${ }^{38}$.

Für die deutsche Literatur ist das 13. Jahrhundert die Zeit der großen Epen geworden:

30) Zu Gerson s. S. RrNGbom, Icon to Narrative. The Rise of the Dramatic Close-up in Fifteenth-Century Devotional Painting, Doornspijk 1984², S. 19 f.

31) Ebda., S. 20.

32) Zu Bild-Wort-Verschleifung im Begriff der Metapher s. W. Harms, Homo viator in bivio. Studien zur Bildlichkeit des Weges ( = Medium Aevum. Philolog. Studien 21), München 1970, bes. S. 153 . - SuCkal.e, Arma Christi (zit. Anm. 28), S. 200, Anm. 32, macht auf die Bedeutung der Nonnenliteratur des 15. Jahrhunderts für dieses Phänomen der Bildersprachlichkeit aufmerksam.

33) D. De Chapeaurougt, "Das Auge ist ein Herr, das Ohr ein Knecht". Der Weg von der mittelalterlichen zur abstrakten Malerei, Wiesbaden 1983, S. 22 f.

34) Zur Privatisierung des Bildes s. Ringbom, Icon to Narrative (zit. Anm. 30), bes. S. $30 \mathrm{ff}$.

35) Zur Kultur der Schriftlichkeit s. H. GrundmanN, Litteratus-illiteratus, in: Arch. für Kulturgesch., 40, 1958, S.1-65; F. H. B.uml, Varieties and Consequences of Medieval Literacy and Illiteracy, in: Speculum, 55,2, 1980, S. 237-265; R. ENGei.sing, Analphabetentum und Lektüre. Zur Sozialgeschichte des Lesens in Deutschland zwischen feudaler und industrieller Gesellschaft, Stuttgart 1973, S. 6-14.

36) BALML, ebda., S. $239 \mathrm{ff}$., dort weitere Literatur. Nach einer Inschrift soll beispielsweise der hl. Ludwig seine Lesekenntnisse von einem Psalterium haben, s. Camul.e, Seeing and Reading (zit. Anm. 24), S. 39.

${ }^{37}$ ) Zum Problem der Volkssprachlichkeit und der Schriftlichkeit s. BAumI., Literacy (zit. Anm. 35), bes. S. 244 ff.

38) CAmul.t., Seeing and Reading (zit. Anm. 24), S. 41. 
Wolfram von Eschenbach, Konrad von Würzburg und Rudolph von Ems schreiben in diesem Jahrhundert ihre Werke. Diese Bewegung vermehrter Lesefreudigkeit, die sich anfänglich noch auf einen engen sozialen Kreis beschränkt ${ }^{39}$, wird, wie der zunehmende private Bücherbesitz im Lauf des 14. und 15. Jahrhunderts belegt, immer breiter ${ }^{40}$. Die Gruppe der teilgebildeten ,illiterati‘, die nun auch illustrierte Werke besitzen, ist nun schichtübergreifend und infolgedessen differenzierter, wodurch die Verfahren, die ihr die teilweise bereits über zweihundert Jahre alten Epen für ganz unterschiedliche Verwendungszwecke nahebringen sollen, entsprechend komplizierter werden.

Um diesen Prozeß zu verfolgen, eignen sich gerade die Illustrationen in volkssprachlichen Texten des 13. bis 15. Jahrhunderts besonders gut, weil hier erstmals die Dominanz liturgischer Bilder gebrochen ist. Deren traditionelle Formengebung wie auch kanonische Inhalte wollten weder dem Betrachter noch dem Hersteller einen großen Bewegungsspielraum für unterschiedliche Interpretationsansätze bieten. Bereits die eingangs besprochenen Beispiele aus dem Trojanerkrieg haben gezeigt, daß vor allem die Illustrationen eine Veränderung durchmachen, werden doch hier fast zur gleichen Zeit, ja manchmal sogar von derselben Werkstatt, sehr diverse Aufarbeitungsmuster angeboten.

\section{Die Illustration als Interpretationsangebot am Beispiel Wilhelms von Orlens}

Um diesen Wandel darstellen zu können, habe ich eines der wenigen Beispiele gewählt, das sowohl in einem relativ frühen illustrierten Exemplar aus dem 13. als auch in Versionen aus dem 15. Jahrhundert überliefert ist. Es handelt sich um einen der beliebtesten Ritterromane, das 1235 von Rudolph von Ems für den Schenken Konrad von Winterstetten geschriebene Epos Wilhelm von Orlens.

Zunächst seien die Gestaltungen des Verhältnisses von Schrift und Bild in der um 1270 entstandenen Münchner ${ }^{41}$ mit der Heidelberger Handschrift von 1420 verglichen $(\text { Abb. } 8,9)^{42}$.

\footnotetext{
39) J. Bumke, Mäzene im Mittelalter. Die Gönner und Auftraggeber der höfischen Literatur in Deutschland 1150-1300, München 1979, hier vor allem zum städtischen Mäzenatentum S. 283 ff.; Dtrs., Höfische Kultur. Literatur und Gesellschaft im hohen Mittelalter, München 1986, S. $675 \mathrm{ff}$. - Zur Situation im Spätmittelalter s. M. L. BAtUMtR, Gesellschaftliche Aspekte der ,Volks'-Literatur im 15. und 16. Jahrhundert, in: Popularität und Trivialitat (= Fourth Wisconsin Workshop), hrsg. von R. Grimm und J. Hermann, Frankfurt 1974, S.6- 19.

40) Zum Beispiel an der Lauber-Werkstatt, s. W. Fechrer, Der Kundenkreis des Dicbold Lauber. In: Zentralbl. für Bibliothekswesen, 55, 1938, S. $121-146$.

41) München, Staatsbibliothek, Cgm. 63; die Handschrift dürfte um 1270 im Elsaß, wahrscheinlich in Straßburg entstanden sein; E. M. VerTtr, Die Bilder, in: Codex Manesse. Die große Heidelberger Liederhandschrift. Kommentar zum Faksimile des Codex Palatinus Germanicus 848 der Universitätsbibliothek Heidelberg, hrsg. von W. KOSCHORRECK und W. Werner, S. 43-88, bes. S. 63 f.; E. J. BEER, Die Buchkunst der Handschrift 302 der Vadiana, in: Kommentarband zur Faksimile-Ausgabe der Handschrift 302 in der Kantonsbibliothek Vadiana St. Gallen, hrsg. von E. J. BtER u. a., Luzern 1987, S. 88; N. H. OTt, Geglückte Minne-Aventiure. Zur Szenenauswahl literarischer Bildzeugnisse im Mittelalter. Die Beispiele des Rodenecker Iwein, des Runkelsteiner Tristan, des Braunschweiger Gawan und des Frankfurter Wilhelm-von-Orlens-Teppichs, in: Jb. der Oswald von Wolkenstein Gesellsch., hrsg. von H.-D. MCCK und U. MCi.I.R, 2,1982/83, S. 1 - 32, bes. S. 15 ff. - Allgemein zu den Wilhelm von Orlens-Handschriften s. M.-M. HARTONG, Willehalm von Orlens und seine Illustrationen, phil. Diss., Köln 1938.

42) Heidelberg, Universitätsbibliothek, Cpg. 323. Die Handschrift ist in der sogenannten Werkstatt von 1418 entstanden; E. Mirti.er und W. Werner, Mit der Zeit. Die Kurfürsten von der Pfalz und die Heidelberger Handschriften der Biblioteca Palatina, Wiesbaden 1986, S. 74; H. WEGtiver, Beschreibendes Verzeichnis der deutschen Bilderhandschriften des späten Mittelalters in der Heidelberger Universitätsbibliothek, Leipzig 1927. S. $19 \mathrm{ff}$.
} 
In beiden Darstellungen ist die Verfolgung der Brabanter durch Wilhelms Vater behandelt, die in der älteren Version mit dessen Tod im Kampf kombiniert ist. Gerade in der Relation der beiden Medien erweisen sich die Blätter als jeweils sehr verschieden. Im Münchner Wilhelm, der auf einer Seite zwei übereinander liegende Bildstreifen anbietet, sind die Bilder als eigene Einheit klar gegen die Schrift abgesetzt. In der Heidelberger Handschrift hingegen stehen sie in einem unmittelbaren Kontakt mit der Schrift, scheint doch das Bild sich um diese herumzulegen. Überdies kommt den roten Überschriften, auf deren Bedeutung noch genauer einzugehen sein wird, eine zwischen Geschriebenem und Gezeichnetem vermittelnde Wirkung zu.

Dieses unterschiedliche Verhältnis wird auch in der Organisation der Bilder selber manifest. So greift in der jüngeren Handschrift das Nebeneinander der Gestalten auf dem sich hinziehenden Terrainstreifen den kursiven Charakter der Schrift auf, ja die von den Blatträndern angeschnittenen Ereignisse wirken wie der zufällige Ausschnitt eines Erzählbandes, bei dem man nur weiterblättern müßte, um die Fortsetzung zu erfahren. Weder Hintergrund noch Rahmen, aber auch nicht andere vereinheitlichende Gestaltungselemente wie Komposition und Farbigkeit wollen in dieser Darstellung eine Konzentration auf ein eigenständiges Bild provozieren. Ganz anders dagegen ist die Anlage in der älteren Handschrift, wo allein der Goldhintergrund hier sehr stark abgerieben - und die Rahmung die doppelzonigen Bilder nachhaltig auszeichnen und sie als besonders hervorgehobene Einheit vom 'Text abgrenzen. Bereits in diesen Grundlagen der Bildsyntax verraten die beiden Versionen jeweils ein ganz anderes Anspruchsniveau: Das doppelzonige Deckfarbenbild mit Gold und Rahmen, zusammen mit der sorgfältigen Schrift und dem feinen Pergament, zeichnen dieses Werk als Luxushandschrift aus. Die Federzeichnung auf Papier dagegen vertritt, in Kombination mit der flüchtigen Bastarda, einen ganz anderen Typus, der vorläufig mit der Etikette „Lesehandschrift" bezeichnet werden soll.

$\mathrm{Daß}$ die so divergierende äußere Präsentation tatsächlich auch für verschiedene Rezeptionsweisen des Werkes bestimmt ist und nicht bloß das Ergebnis zufällig vorhandener Materialien oder gar - wie Werner Schröder meint ${ }^{43}$ - als Mißverständnis und Zweckentfremdung des Werkes gedeutet werden muß, ist nun mit eingehenderen Analysen zu belegen. Zunächst soll am Münchner Beispiel, also der älteren Handschrift, gezeigt werden, daß mit dem formalen Anschluß an die Tradition lateinischer, illustrierter Handschriften nicht allein Ausstattungsgewohnheiten übernommen werden, sondern damit auch der Bedeutungshintergrund der Vorbilder übertragen wird.

Der Typus des doppelzonigen Bildes mit Hintergrund und Rahmen ist im Bereich der lateinischen Handschriften, deren ausschließliche Dominanz wir ja gerade in solchen Stücken wie dem volkssprachlichen Münchner Wilhelm erstmals durchbrochen sehen, vor allem in Psalterien überliefert ${ }^{44}$. Ferner gehört er zur Prunkausstattung narrativer Zyklen, unter denen die in Paris um 1240 entstandene Bibel der Pierpont Morgan Library (Abb. 11) zweifellos der bedeutendste ist $^{45}$. In der deutschen Epenhandschrift - etwa in der Verfolgung Jofrits und dem Tod von Wilhelms Vater (Abb. 8) - ist das Dekorationssystem des französischen Werkes in vereinfachter Form zu finden. Darüber hinaus sind - ohne daß eine direkte Abhängigkeit postuliert würde - wichtige Elemente der Bildstruktur übernommen worden. Vergleichen wir etwa den Kampf,

\footnotetext{
43) W. SCHRODER, Literatur, Literaten und Laien im deutschen Spätmittelalter. Revue eines interdisziplinären Rundgesprächs, in: Mittellat. Jb., 20, 1985, S. 216-223, bes. S. 217.

44) Zu dieser Uberlieferung s. Beer, Handschrift 302 (zit. Anm. 41), S. 114 f.

45) New York, Pierpont Morgan Library, M 638; Abb. s. S. C. Cockerei.1., Old Testament Miniatures, New York 1969.
} 
den Wilhelms Vater mit Jofrit durchficht (Abb. 10), mit dem alttestamentlichen Kampf Davids gegen die Philister, so verwendet die deutsche Handschrift nicht allein Rahmen und Hintergrund, sondern vor allem die Organisation der Formen innerhalb des Bildfeldes in ganz verwandter Weise. In beiden Beispielen ist der Hintergrund Folie, Bildfeldbegrenzung, vor der in äußerst schmalen Schichten die verschiedenen Ebenen der Erzählung voreinander gelegt werden. Der Brennpunkt der Erzählung dient meist als Rotationspunkt für die gesamte Komposition. Mit dem Mittel des Übereinanderschichtens von nahezu heraldisch gewordenen Formen, die an einem bestimmten Punkt gebündelt werden, ist eine Wucht, Vielschichtigkeit und zeitliche Verschränkung der Kämpfe erreicht, die das Epos in seinen Darstellungen den alttestamentlichen ebenbürtig macht.

$\mathrm{Da}$ es sich hier nicht bloß um eine formale Angleichung der Bildersprache handelt, sondern damit auch eine Bedeutungsübertragung stattgefunden hat, wird durch die Verwandtschaften motivischer Art unterstrichen. Eine der beliebtesten Pathosformeln in der Bibel wie im Epos ist der Ausritt der Helden. Wilhelm zieht genauso in die Bewährungskämpfe - hier zum Kampf um Galverne (Abb. 12) - wie David gegen die Amalekiter (Abb. 13): Beide Helden sind hier ebenbürtig mit Troß und Bewaffnung in einer Situation geschildert, die auch ihre Gemeinsamkeit betont, nämlich die Erfüllung ritterlicher Pflicht in der Aufnahme des Kampfes.

Im weiteren soll nun belegt werden, daß in der Münchner Version des Wilhelm dieser Bezug zur lateinischen und damit auch religiösen Tradition weit mehr als eine bloße Analogie von Formen und Motiven ist, sondern überhaupt das zentrale Aneignungsraster für diesen Text darstellt. Hierfür muß das Bildprogramm in die Argumentation miteinbezogen werden. Allerdings ergibt sich für diesen Analyseschritt eine besondere Schwierigkeit: Bisher habe ich es vermieden, den Inhalt des Wilhelm-Epos zu schildern, weil dies mit einer zentralen Problematik unseres Themas zu tun hat. Jede Inhaltsangabe des Romans ist bereits eine interpretierende, die - geht sie einzig vom Text aus - mit den ganz anders gelagerten und außerordentlich divergierenden Sinnschichten, welche die Bilder den einzelnen Wilhelmhandschriften beifügen, nicht übereinstimmen kann: Es müßte infolgedessen von den Bildern eine jeweils ganz anders lautende Inhaltsangabe erfolgen. Mit dem Münchner Literarhistoriker Norbert Ott möchte auch ich von der Annahme eines ,,autonomen Werkes“ abrücken, da dieses nur als ein immer wieder neu verstandenes, für ganz unterschiedliche Funktionen neu interpretiertes gesehen werden $\mathrm{kann}^{46}$. Dies bedeutet, daß das Werk uns meist als rezipiertes überliefert ist, was besonders deutlich wird bei Abschriften des 15. Jahrhunderts, wo ja bereits über zweihundert Jahre Abstand zur ursprünglichen Autorintention bestehen. Für unsere Problematik hat eine solche Prämisse weitreichende Folgen, ist uns doch damit jeglicher Anspruch, über die Richtigkeit des Stellenwertes der Bilder zu urteilen, aus der Hand geschlagen. Wir können zwar feststellen, daß Ereignisse fehlen, die uns vom Ablauf der Geschichte oder auch von der Konstruktion des Werkes her als zentral erscheinen, wogegen andere, nur am Rande erwähnte, vielleicht im Text nur mit einem Wort angedeutete Ereignisse manchmal ganze Bildsequenzen auslösen. Diese andere Gewichtung läßt sich nicht qualitativ beurteilen und etwa als mangelhafte oder mißverstandene Textüberlieferung abtun, sondern muß als Strukturprinzip analysiert werden, das programmatische Anliegen ausdrückt. Primär muß sie konsequenterweise als sinngebend aufgefaßt werden, auch wenn das unserem Literaturbild noch so sehr zuwiderlaufen sollte. Dies bedeutet, daß die Bilder die Leserichtung des Textes, seine inhaltliche Gewichtung mitbestimmen

${ }^{46}$ ) OTT, Uberlieferung (zit. Anm. 23), S. 357. - Dagegen vgl. SCHródtr, Literatur (zit. Anm. 43), S. 217. 
und wir erst aus ihnen erschließen können, ob ein bestimmtes Werk etwa als "Staatsroman“ mit heilgeschichtlichen Bezügen ${ }^{47}$, als ein Lehrbuch für Verhaltensformen im städtischen Bereich oder als ein Minneroman aufgefaßt werden sollte.

In unserem Zusammenhang des Wilhelmepos soll als Kontrast an ein Beispiel derselben Thematik aus einem ganz anderen Medium erinnert werden, an den Wilhelm von Orlens-Teppich aus dem frühen 15. Jahrhundert, der im Frankfurter Kunstgewerbemuseum aufbewahrt wird (Abb. 18): Allein im ersten Viertel des Teppichs zeigt hier die Bilderauswahl eine völlig andere Gewichtung des Epos als sie uns bisher bekannt ist: Schachspiel, Abschied von der Geliebten, doppelte Darstellung von Minnekrankheit und Heilung durch die Angebetete leiten ein zu einer unablässigen Folge von Minneabenteuern und Bewährungstaten um die Geliebte ${ }^{48}$. Eine solche Interpretation findet sich m. E. in keiner der illustrierten Wilhelm von Orlens-Handschriften, deren Bilderzyklen wesentlich vielschichtiger aufgebaut sind. Ohne daß hier nun die Programme in ihrer Gesamtheit besprochen werden können, was für eine genaue Analyse unbedingt erforderlich wäre, soll versucht werden, die Unterschiede der Interpretationsansätze an einer bestimmten, in allen Handschriften als zentral angesehenen Stelle herauszuarbeiten, nämlich am Beispiel der erstmaligen Präsentation und Anerkennung des Helden Wilhelm im Bild.

Keine Bedeutung wird in den Illustrationen der Geburt Wilhelms zugemessen, welche im Text (V. 1632), in unmittelbarer Fortsetzung der Genealogie, im Moment des väterlichen Todes erfolgt ${ }^{49}$. In der Münchner Version (Abb. 14) wird das Motiv der genealogischen Kontinuität allerdings in einer sehr kompakten Bildaussage angesprochen. Diese ist gerade mit ihren vielfältigen Bezugssystemen ein gutes Beispiel für den ganz andersartigen Interpretationsvorgang des Bildes im Vergleich zum Text: In dieser Darstellung wird der Knabe erstmals im Bild eingeführt. Er wird von einer Frau in den Armen präsentiert, die an der Totenbahre seines Vaters steht, über der seine Mutter Ilye, den Minnetod erleidend, zusammenbricht. Illustriert wird die folgende Stelle (V. 1932): Sie bot irn roten munt / sim (vater) bobet dik unde dike / ... drubte si an sich den lip, darauf bricht ihr das Herz, Blut rinnt ihr zu Mund und Nase heraus und (V. 1946): Also lac si bi im tot. Die Darstellung in der Handschrift in Den Haag (Abb. 15) aus dem mittleren 15. Jahrhundert betont, ganz im Gegensatz zu München, gerade diese drastische Schilderung des mütterlichen Todes und schildert mit besonderer Textgenauigkeit das Zusammenbrechen der vor Schmerzen Blutenden ${ }^{50}$. Daß die Münchner Handschrift eine ganz andere Interpretation der betreffenden Stelle liefert, belegt schon ihr Zusammenspiel mit weiteren Szenen, die kurz interpretiert werden sollen. Die liturgische Handlung, die neben der Einführung des Helden im Moment des Todes seiner Eltern erfolgt, illustriert eine wesentlich frühere Textstelle. In den Versen 1807ff. nämlich wird von der Messe berichtet, die Ilye zu Ehren ihres Mannes lesen läßt. Erst danach besteht sie darauf, den Leichnam des Gatten zu sehen und bricht - wie im Bild links geschildert - über seiner Bahre zusammen. Die Messe des Textes wird hier, der Leserichtung des Bildes folgend, als paralleles oder sogar späteres Ereignis erfahren und dient als allgemeine liturgische Handlung der Überleitung vom oberen zum unteren Bildstreifen. In diesem wird die Taufe des Helden in der gesamten Bildbreite geschildert und nimmt

47) Zum Begriff Staatsroman s. OTt, Weltchronik-Ikonographie (zit. Anm. 23), S. 31 f., dort weitere Literatur.

48) OTt, Minne-Aventiure (zit. Anm. 41), S. 14-18; Abb. des gesamten Teppichs s. D. Heinz, Europäische Wandteppiche, Braunschweig 1963, Abb. 108.

${ }^{49}$ ) V. Junk, Rudolfs von Ems Willehalm von Orlens (= Deutsche Texte des Mittelalters, Bd. 2), Berlin 1905.

5) Den Haag, Koninklijke Bibliotheek, Ms. 76 E 1; die Wasserzeichen datieren von 1448-1450; s. Schatten van de Koninklijke Bibliotheek, Ausst. S'Gravenhage 1980, S. 129, dort weitere Literatur. 
als Ereignis, das im Text (V. 2055) relativ nebensächlich und erst nach dem feierlichen Begräbnis der Eltern (im Bild weggelassen) erwähnt wird, die prominenteste Stelle ein. In dieser Doppelanlage also ist ein Zusammenzug ganz verschiedener Textstellen vorgenommen, die zugleich eine neue Deutung erfahren haben: Die genealogische Legitimität aus dem Doppeltod ist gewährleistet, aber das Auftreten des Helden wird gänzlich eingebettet in den liturgischen Akt der Taufe, womit seine Christlichkeit in einem Maße betont wird, wie es der Text gar nicht anstrebt.

Darüber hinaus vermag aber das Bild noch weitere Deutungsschichten zu eröffnen, die wiederum über die Allusion an große ikonographische Vorbilder evoziert werden. Die aus Liebe zu ihrem verstorbenen Mann zusammenbrechende Ilye wird hier in Analogie gesetzt mit Maria, "die das Haupt und die Schultern des toten Sohnes umfaßt", wie es in den Meditationes Vitae Christi zur Totenklage Mariens ${ }^{51}$ heißt. Dieser Topos der reinen Liebe und Trauer kumuliert bekanntlich in den Beweinungsbildern, einem Bildtypus, der in der nordischen Malerei des 13. Jahrhunderts noch keineswegs üblich ist und erst im 14. Jahrhundert als Andachtsbild eine größere Verbreitung findet. Der wohl auch in Straßburg entstandene, eine Generation ältere Bonmont-Psalter (Abb. 16) kennt bei der Darstellung der Grablegung Christi eine sehr verwandte Konstellation ${ }^{52}$. Dasselbe Prinzip der Übertragung vermochte überdies Beer um 1300 festzustellen in der hochrheinischen Version der Karls-Vita in St. Gallen ${ }^{53}$, die bereits vom Text her als „Staatsroman" konzipiert ist. Hier wird die Ikonographie auf den Tod Rolands transferiert und damit ebenfalls eine Parallelisierung mit dem Tod Christi angestrebt.

Der Vorstellungskreis der ungebrochenen Genealogie, im Motiv der Ablösung des Todes durch das Leben des neuen Helden angesprochen, ist im Wilhelm mit einem weiteren heilsgeschichtlichen Topos in Zusammenhang gebracht. Der junge Wilhelm in den Armen einer die Bahre der hinscheidenden Person begleitenden Gestalt wird üblicherweise als Formel für die Wiedergeburt der Seele Mariens in den Armen des Herrn gebraucht. Zum Vergleich sei hier wiederum an oberrheinische Beispiele erinnert, wie an den Marientod im Wonnentaler Graduale oder - noch näher - im etwas älteren Prozessionale (Abb. 17) des Freiburger Stadtarchivs ${ }^{54}$. Auf die besondere, heilsgeschichtliche Bedeutung der Taufszene im Szenenzusammenhang weist die Konzeption des Blattes insgesamt hin, wird ihr doch die ganze untere Bildzone eingeräumt. Das Motiv der Taufe nun gehört zum festen Kanon eines jeglichen Heiligenzyklus ${ }^{55}$ und weist auf den entscheidenden Wandel hin von einer üblichen Lebensbeschreibung zur christlichen Vita.

5t) G. Schil.ter, Ikonographie der christlichen Kunst. Bd. 2, Gütersloh 1966, S. 189.

52) Besançon, Bibliothèque Municipale, Ms. 54; s. Ausst.-Kat. Die Zeit der Staufer, Ausst. Stuttgart 1977, Bd. 1, Nr. 723; S. 543-545, dort weitere Literatur.

53) Bter, Handschrift 302 (zit. Anm. 41), S. 80.

54) Abb. zum Wonnetaler Graduale s. E. J. BeER, Die Buchkunst des Graduale von St. Katharinenthal, in: Kommentarband zur Faksimile-Ausgabe hrsg. von L. W ITrerich u. a, Luzern 1983, Abb. 25. - Freiburg, Stadtarchiv, Hs. 122; Literatur s. C. GotTw AI.D, Die Musikhandschriften der Universitätsbibliothek und anderer öffentlicher Sammlungen in Freiburg im Breisgau und Umgebung (= Kataloge der Universitätsbibliothek Freiburg im Breisgau, hrsg. von W. KeHr, Bd. 1, Teil 2), Wiesbaden 1979, S. $117 \mathrm{f}$.

55) Etwa Taufe Konstantins im Stavelot-Triptychon, Abb. W. Votikj.E, The Stavelot Triptych. Mosan Art and the Legend of the True Cross, New York 1980, Taf. 4; Vita des Hl. Dionys, Abb. s. R. BranNer, Manuscript Painting in Paris During the Reign of Saint Louis, Berkeley 1977, Abb. 262. - Die Plazierung der Taufe der HI. Margarethe ins Zentrum eines Stickereiteppichs aus dem späten 13. Jahrhundert in Helmstedt belegt dic Bedeutung, die dieser Szene zugemessen wird; Abb. s. R. Kroos, Niedersächsische Bildstickereien des Mittelalters, Berlin 1970, Abb. 122. 
Fassen wir die Ergebnisse der Analyse am Münchner Wilhelm zusammen, so hat sich sowohl vom Bildtypus und seiner Syntax als auch von seinem Assoziationsfeld her eine weitgehende Lösung vom Text zugunsten einer völlig neuen Interpretation erkennen lassen. Der Roman wird heilsgeschichtlich interpretiert, die Ereignisse sind so ausgewählt, daß sie einem typologischen Muster entsprechen und daß der scheinbar profane Stoff seine Überhöhung als Exempel göttlicher Offenbarung findet. Das Epos wird dadurch zum „Staatsroman“, in dem die Macht des Helden und - in Wilhelms Fall erst gegen Ende der Geschichte - die des Herrschers heilsgeschichtlich legitimiert werden, womit eine Interpretation des Wilhelm-Stoffes gegeben ist, die unserem üblichen Verständnis des Textes keineswegs entspricht.

Einer wiederum ganz anderen Konzeption begegnen wir im Stuttgarter Exemplar, dem wohl am reichsten illustrierten Wilhelmzyklus ${ }^{56}$. Die Papierhandschrift ist in derselben Werkstatt wie die Heidelberger Version, mit der sie das eingangs besprochene, besondere Verhältnis des Bildes zur Schrift teilt, im Jahr 1419 wohl in Colmar oder Straßburg hergestellt worden. Im Gegensatz zur Münchner Ausgabe stehen auch hier - etwa in der Darstellung des aus der Stadt getragenen Leichnams von Wilhelms Vater (Abb. 19) - Bild und Schrift in unmittelbarem Kontakt: Das Bild benutzt den beiden Medien gemeinsamen Blattrand und legt sich damit um den Textblock, zugleich unterstützt es in seiner eigenen Konzeption deutlich die Leserichtung. Die rubrizierten Tituli verstärken zudem die enge Beziehung zwischen den beiden Medien, dienen sie ihnen doch als Verbindungsglied.

Überschriften nun sind ein wichtiges und neues Charakteristikum all dieser Codices aus der ersten Hälfte des 15. Jahrhunderts. Ihrer Funktion nach können sie sowohl Bild- als auch Kapitelbezeichnungen sein und tragen offenbar primär zur Gliederung des Buches in besondere Lese- und Bildabschnitte bei. Damit nehmen sie eine Vermittlerrolle ein, können sie doch sowohl zum Text als auch zum Bild gehören. Sie sind nun aber nicht bloß Lesezeichen, sondern interpretieren ihrerseits den Text und legen, zumal in den Handschriften des mittleren 15. Jahrhunderts, weitgehend auch das Thema für den Maler fest ${ }^{57}$. In diesen Fällen muß also die Art der Überschrift - ihr Verhältnis zum Text und zum Bild - abgeklärt werden, um überhaupt den Bezug der Illustration zu diesen zwei schriftlichen Medien eruieren zu können.

Prüfen wir nun am Stuttgarter Exemplar dieselbe Textstelle, mit der wir uns schon im Münchner Wilhelm beschäftigt haben: Das Eintreten des Helden in die Bilderzählung. Zunächst ist festzustellen, daß die in der Münchner Version in einem Blatt zusammengezogenen Passagen hier auf mindestens neun, dem Fluß des Textes folgende Bilder verteilt sind ${ }^{58}$. Das bedeutet, daß auch das Illustrationsprogramm, wie bereits beim Bildtyp vermutet, den Lesezug betont.

${ }^{56)}$ Stuttgart, Württembergische Landesbibliothek, Cod. H B XIII 2; die Handschrift ist 1419 von Johannes Coler geschrieben und in der Werkstatt von 1418 mit Illustrationen versehen worden; M. S. BUHI. und L. KURAS, Die Handschriften der ehemaligen Hofbibliothek Stuttgart, 4,2, Wiesbaden 1969, S. $80 \mathrm{f}$.

$\left.{ }^{57}\right) \mathrm{Zu}$ dieser weitgehenden Orientierung an den Überschriften und deren Bedeutung für die Bildinhalte s. L. E. Stamm-Saurma, Die Illustrationen zu Konrads Trojanerkrieg, in: Das ritterliche Basel. Zum 700. Todestag Konrads von Würaburg, Ausst. Basel 1987, S. 62-68, bes. S. 66f. - Zum generellen Problem der Tituli als strukturell vermittelndes Medium zwischen Wort und Schrift s. M. Camil.e, The Book of Signs, Writing and visual difference in Gothic manuscript illumination, in: Word and Image, 1/2, 1985, S. 133-148.

58) Die Einführung des Helden wird in folgenden Darstellungen thematisiert: fol. 33v: Wilhelms Tod wird an seinem Sarg beklagt, Frau Ilye sinkt darnieder; 37: Wilhelms Untertanen schwören dem Kind auf den Armen Ilyens den Eid; 39v: Frau Ilye wird vom König von Frankreich zur Messe geführt; 40v: Frau Ilye beklagt den Toten und folgt ihm nach; 43v: Frau llye und Wilhelm werden beklagt, der König von Frankreich hält das Kind; 45: Taufe Wilhelms, der König von Frankreich anerkennt ihn; 51, 53v: Boten zwischen Jofrit und König Philipp; 56: Eid vor dem Kind. 
Betrachten wir nun die erste Präsentation des Helden (Abb. 20), so sieht diese ganz anders aus als die entsprechende Szene der Münchner Handschrift. Ihre Beischrift, die auf der gegenüberliegenden Seite angebracht ist, lautet folgendermaßen: wie frowe ylie ir volck besante und ir biesz botschaft bringen von wilbelm von orlenz und sü sich übel gebuop und wie sü dem kinde swuren. Dieser Titulus stellt eine nahezu vollständige Zusammenfassung der Verse 1645 - 1720 dar und referiert, Worte des Textes aufnehmend, die Ereignisse dieser Passage. Im Gegensatz zur älteren Version wird hier also, zumindest in den Überschriften, Wert auf eine Übersicht über die im Text geschilderten Abläufe gelegt. Anders allerdings verfährt das dazugehörige Bild. Darin sind nur die Verse 1718 bis 20 gestaltet, wo es heißt: dez warent si ir vil berait/ und swuorent dem kinde do / bulde. Denn dargestellt sind hier nicht die im Titulus erwähnten Ereignisse, wie Frau Ilye vom Tode Wilhelms erfährt und in Trauer fällt, sondern lediglich das Schwören des Volkes. Hochaufgerichtet bietet die Königin den zur Klärung seiner Stellung bereits bekrönten kleinen Wilhelm den Herren zum Treueschwur dar. Erschütternde Ereignisse, wie die Überbringung der Todesnachricht und die emotionale Reaktion der Gattin, erfahren hier eine deutliche Zäsur durch die prominente Darstellung des juridischen Aktes, in dem der Held seine Anerkennung erfährt. Dem Text zuwiderlaufend sind überdies die Untertanen nicht als bloßes Volk gekennzeichnet, sondern sind in ihrer gesellschaftlichen Situation klar definiert. Ihre Kleidung läßt sie als vornehme Städter erkennen. Schellenkette, weit geschlitzte und gezaddelte Mäntel, die Kappe mit den Wollkordeln und der kurze Männerrock sind hier so dargestellt, wie sie eine zeitgenössische Quelle als besonders modisch beschreibt ${ }^{59}$.

Vergleicht man diese den Helden einführende Szene mit der Münchner Handschrift, so ist der Unterschied eklatant: War dort die Überhöhung Wilhelms als ,miles christianus' in einem allgemein ,heiligen ' Kontext das zentrale Thema, so ist hier seine Einordnung in einen klar benennbaren sozialen Rahmen gesucht. Weder das Motiv der genealogischen Abfolge noch die Trauer oder der Minnetod der Mutter sind hier berücksichtigt, sondern es geht einzig um die rechtliche Anerkennung des Helden durch den Treueeid, den Angehörige einer Oberschicht leisten.

Diese am Einzelbild zu beobachtende Tendenz stellt sich bei der Programmanalyse der Illustrationen als das bestimmende Prinzip heraus. So wird etwa das allmähliche Heranwachsen Wilhelms in eine Sequenz von immer neuen Schritten gesellschaftlicher Legitimation aufgelöst. Nur widerwillig schwören dem kleinen, von Jofrit erzogenen Waisen (Abb. 21) die Treue ,Volk und Herrschaft", beide als wenig differenzierte Gruppe dargestellt, die nur allgemein auf eine Oberschicht verweist. Darauf folgt die Szene (Abb. 22), wie Wilhelm als junger Mann vom Kaiser das Fahnlehen erhält; ein Stab mit der Fahne wird ihm übergeben, womit Wilhelms Erhebung in den weltlichen Fürstenstand dokumentiert ist. Die Bedeutung dieses Ereignisses wird sogar noch durch eine Wiederholung unterstrichen, insofern darauf die Zeremonie der Verleihung des Lehensbriefes folgt (Abb. 23). In beiden Darstellungen besonders betont sind die genauen Hinweise auf die gesellschaftliche Stellung der Kontrahenten, wie etwa Reichskrone und Thron des Kaisers, und der Rechtsvorgang ist jeweils mit einer Korrektheit geschildert, $\mathrm{da} ß$ er in dieser Form durchaus den Illustrationsanforderungen einer juristischen Handschrift, etwa in der Art der Sachsenspiegel, genügen dürfte ${ }^{60}$.

${ }^{59}$ ) A. Scrul.z, Deutsches Leben im XIV. und XV. Jahrhundert, Wien 1982, Bd. 1, S. 312.

60) Eine ikonographisch identische Darstellung des Fahnlehens findet sich im Sachsenspiegel, Abb. s. W. Ko. SCHORRECK, Der Sachsenspiegel in Bildern, Frankfurt 1976, Nr. 10. 
Versuchen wir nun, diesen Befund zu deuten und die Funktion der Bilder im Stuttgarter Programm zu eruieren. Titulus und Bild greifen hier besonders eng ineinander, indem der Titel einer linear raffenden Wiedergabe der wichtigsten Textabschnitte dient und das Bild in einer ersten Sinnschicht davon eine weitere Zusammenfassung liefert. Beide Medien erleichtern somit insofern den Lesevorgang, als sie dem Benützer den Text in überschaubare Abschnitte vorbereiten. Der leichteren Lesbarkeit kommt übrigens auch der Text selber entgegen, der in das Idiom eines bestimmten Benützerkreises übertragen ist ${ }^{61}$. Der Absicht, Wissen und Materie an ein Publikum zu vermitteln, das erst mit dieser relativ neuen Art volkssprachlicher Schriftlichkeit gewonnen werden kann, entspricht auch die Wahl der Kategorie der Federzeichnung. Sie ist nämlich in der traditionellen Praxis der Buchausstattung vor allem didaktischen Handschriften vorbehalten gewesen.

Mit der Problematik der neuen, weiteren Verbreitung der Schriftlichkeit dürfte auch der besondere Schwerpunkt dieses Programms zusammenhängen, nämlich die extreme Betonung aller Akte, die mit dem geschriebenen Wort zu tun haben. Die unzählige Male wiederholten Darstellungen des Schreibens und Übergebens von Geschriebenem, beispielsweise Wilhelm einen Brief an seine geliebte Amelie schreibend (Abb. 24), scheinen das besondere Gewicht des geschriebenen Wortes zu betonen, seine Richtigkeit geradezu zu verbriefen. Damit verbunden muß noch eine zweite Sinnschicht gesehen werden, umfaßt das Programm doch eine fast ausschließliche Sequenz juristischer Umgangsformen zwischen den einzelnen Ständen. Liebesszenen und Bewährung im Kampf, die ja beispielsweise in dem nahezu gleichzeitigen Frankfurter Teppich so sehr im Vordergrund stehen, werden hier peripher behandelt. Szenen juristischer Art im weitesten Sinn dagegen, die in den anderen Versionen meist nicht beachtet und im Text oft gar nicht explizit erklärt werden, sind hier in allen Einzelheiten ausgestaltet, ja nicht selten sind bloße Gespräche zu solchen Akten umdefiniert. So ist etwa die Unterredung von Wilhelms Vater mit seinen Mannen vor der entscheidenden Schlacht zu einer Ratssitzung ausgebaut (Abb. 25) und der Schwur Wilhelms vor Amelie (Abb. 26) wird gar zur wichtigsten Szene der gesamten Begegnung zwischen den beiden Liebenden. Daß mit einer solchen Auswahl der Wilhelmroman zu einem Lehrbuch juristischer Verhaltensmodi zwischen Personen verschiedenen Standes, Geschlechts und Alters wird, scheint unzweifelhaft ${ }^{62}$.

Schwieriger ist die Beantwortung der Frage nach der Funktion, die ein solches Interpretationsmuster zu erfüllen hatte. Ein Rückschluß anhand etwas späterer, verwandt strukturierter Programme dürfte hier einige Anhaltspunkte liefern. Diese können allerdings nur in eine ungefähre Richtung weisen, da gerade im 15. Jahrhundert ein sehr starker Wandel von Gebrauchssituationen zu beobachten ist und nicht selten scheinbar verwandte Interpretationen sich bereits

\footnotetext{
61) Daß in diesen Ateliers offenbar auch Dialektabschriften für bestimmte Benutzer hergestellt werden können, belegt die Historienbibel in Kopenhagen, Königliche Bibliothek, Ms. Thott. $1232^{\circ}$, die in der Lauber-Werkstatt ausgestattet, aber in ripuarischem Dialekt offensichtlich für den Käufer, Johann von Nassau, abgefaßt ist: L. E. Stamm, Auftragsfertigung und Vorratsarbeit, in: Unsere Kunstdenkmäler, 36, 1985, S. $301-309$.

${ }^{62}$ ) Wehrli interpretiert den Roman als „eine Art Fürstenbiographie ... hat den Sinn beinahe eines Lehrbuches des richtigen höfischen Verhaltens, vom Minnedienst und der Hofetikette bis zum Waffenhandwerk in Sport und Krieg, vom Briefeschreiben bis zur politischen Verhandlung, von der Finanzverwaltung und dem Recht bis zur feinen Küche ... “, s. M. Wt.HRL., Geschichte der deutschen Literatur vom frühen Mittelalter bis zum Ende des 16. Jahrhunderts, Bd. 1, Stuttgart 1984², S. 487 f. - Von diesem breiten Angebot scheinen auch die Maler der Stuttgarter Handschrift nur einen selektiven Gebrauch zu machen.
} 
nur eine Generation später an einen anderen Zusammenhang richten können ${ }^{63}$. Im zweiten Drittel des 15. Jahrhunderts läßt sich bei den Illustrationen des Belial, einem Text, welcher die Legitimierung der Rechtspraxis am Beispiel eines Satansprozesses anstrebt, ein ähnliches Programm beobachten. Für jede Prozeßstation wird mit der Schriftlichkeit die Richtigkeit des Verfahrens bewiesen. In einer Darstellung etwa der Wiesbadener Handschrift (Abb. 27) aus der späten Lauber-Werkstatt ${ }^{64}$ wird der Urkundenbeweis, die Rechtsgültigkeit des schriftlichen Wortes also, durch nichts Geringeres als durch die Bibel belegt. Diese wird hier dem Richter überreicht, damit er sich auch des Rechts versichere. Wie Norbert Ott nachweisen konnte ${ }^{65}$, ist gerade die Schriftlichkeit für die Berufsstände, die mit juristischen Belangen zu tun haben, von besonderer Bedeutung, und die Handschriften scheinen denn auch vor allem für Notare hergestellt worden zu sein. Die strukturelle Verwandtschaft im Bildprogramm der Stuttgarter Wilhelmhandschrift läßt einen ähnlichen Rezeptionszusammenhang wie beim Belial vermuten. Auch hier weisen gerade die Betonung der Verwaltungsakte und die Bestätigung ihrer Richtigkeit durch die Schrift darauf hin, daß der Text für einen Benützerkreis aufgearbeitet wurde, der ebenfalls mit diesen Tätigkeiten befaßt war. Naheliegend wäre es hier, etwa an ein städtisches Ratsherrenpublikum zu denken.

Versuchen wir nun, die bisherigen Resultate des Vergleichs zwischen den beiden Wilhelmhandschriften anhand meiner eingangs aufgestellten Hypothesen zu prüfen: Der Unterschied zwischen den zwei Werken hat die Annahme einer Zusammengehörigkeit beider Medien bestätigt. Die Anpassung der Idiome und die Gliederung des Textes in überschaubare Einheiten wie auch die Wahl von Bildtyp und Programm in der jüngeren Handschrift haben den Wandel des Verhältnisses zur volkssprachlichen Schriftlichkeit und damit auch der Lesegewohnheiten reflektiert. Während das Münchner Exemplar sich ganz aus der lateinisch-liturgischen Tradition ableitet, stellt der Stuttgarter Codex offenbar einen ersten Schritt zur Emanzipierung volkssprachlicher Schriftlichkeit dar. Was die zweite Hypothese betrifft, so hat sich die Annahme der größeren Anpassungsfähigkeit des Bildes vor allem bei der Stuttgarter Version bestätigt. In diesem Beispiel gelingt es der Illustration in einem ganz anderen Maß als dem tradierten Text, dessen Belange in einer dem Betrachter vertrauten Welt anzusiedeln. Instrumente dieser Interpretationen sind, wie die Analysen gezeigt haben, Bildsyntax und Programm, die im älteren Manuskript den Wilhelmroman als „Staatsroman“ anboten, wogegen er im jüngeren zum juristischen Lehrbuch für eine städtische Oberschicht wird. Zum dritten Punkt: Trotz der unzweifelhaft geringen künstlerischen Qualität der Stuttgarter Illustrationen vermögen diese Fe-

63) Die Handschrift in Den Haag jedenfalls (vgl. Anm. 50) stimmt, obwohl sie eine ältere, wahrscheinlich sogar in der Werkstatt von 1418 entstandene Handschrift als Vorlage gehabt haben dürfte, mit ihrer Interpretation keineswegs mehr mit den Vorgängerwerken überein. Hier schafft die Art der Bilderzählung trotz dem scheinbar ähnlichen Programm eine völlig neue Textauslegung, die auch mit einer anderen Art der Verwendung und vielleicht sogar einem anderen Benutzerkreis rechnet: Die Handschrift ist wohl im Auftrag Katharinas von Österreich hergestellt worden; s. Ausst.Kat. Boeken van en rond Willem van Oranje, Ausstellung S'Gravenhage Koninklijke Bibliothcek 1984, S. 26.

64) Wiesbaden, Hessische Landesbibliothek, Hs. 66; s. N. H. ОтT, Rechtspraxis und Heilsgeschichte. Zur Uberlieferung, lkonographie und Gebrauchssituation des deutschen „Belial“ (= Münchner Texte und Untersuchungen zur dt. Lit. des Mittelalters, Bd. 80), München/Zürich 1983, S. 334-337. - Auf die Verwandtschaft der Stuttgarter WilhelmInterpretation zu den späten Belialhandschriften machte mich Norbert Ott aufmerksam, dessen Hilfe und Anregungen dieser Artikel viel verdankt.

65) OTT, ebda., S. 254. 
derzeichnungen angesichts veränderter Bedürfnisse den Anforderungen einer Neuinterpretation des Textes durchaus mit vergleichbarer Effizienz gerecht zu werden.

Zum Abschluß soll die Problematik einer übergreifenden Theorie zum Verhältnis von Illustration und Text am Beispiel einer dritten Wilhelmhandschrift wenigstens noch angesprochen werden. Zu leicht könnte nämlich jetzt der Eindruck entstehen, wir hätten in den zwei besprochenen Varianten die beiden einzig möglichen vor uns, die es nun zu verallgemeinern gelte. Ein ganz anderes Modell etwa liefert der Wilhelmzyklus in der schon bekannten Handschrift von 1441 in Nürnberg ${ }^{66}$. Bei diesem Sammelband handelt es sich gleichsam um ein Kraftpaket von Ritterromanen, ist doch darin der Trojanerkrieg des Konrad von Würzburg und der Herzog Ernst mit dem Wilhem von Orlens zusammengebunden. Trojanerkrieg und Wilhelm sind beide nach demselben Muster illustriert, das am Epos des Rudolf von Ems kurz vorgestellt werden soll. Wilhelms Verlust der Eltern, seine Erziehung und die Jugendliebe zu Amelie werden in wenigen Bildern zusammengefaßt ${ }^{67}$. Erst im Moment der Feiern zu Ehren seines Ritterschlages wird der Zyklus intensiv und Wilhelm als aktive Figur eingeführt. Nach der Begrüßung durch Jofrit (fol. 223) und der Übergabe von Amelies Brief durch Pittipasz (fol. 225v) folgt eine dichte Sequenz ritterlicher Kämpfe, in deren Zentrum Wilhelm steht. Diese Darstellungen sind mit ungewöhnlicher Präzision wiedergegeben und unterscheiden die verschiedenen Varianten ritterlicher Kämpfe scharf voneinander. So nimmt Wilhelm zweimal an einem Kolbenturnier (Abb. 28) teil, während in den anderen Darstellungen entweder ein Rennen oder ein scharfes Gestech (Abb. 30) festgehalten sind. Die im weiteren geschilderten Ereignisse muten an, als ob sie eine Dokumentation des in jener Zeit üblichen Ablaufs einer Turnierveranstaltung wären. Damit entspricht dieses Illustrationsprogramm dem Typus der Turnierbücher, die nur kurze Zeit später als neue Gattung aufkommen ${ }^{68}$. So sitzt etwa die Turnierkönigin mit dem Falken in der Hand unter den Frauen des weiblichen Ehrengerichts (Abb. 29), während Wilhelm, wie es seinem Fürstenstand geziemt, von vier Turnierknechten begleitet, unter denen einer als Grogier dient, in die Kämpfe zieht. Anschließend folgen verschiedene Zweikämpfe und Festessen. Die Illustrationen gipfeln schließlich, ohne je den Faden der Geschichte zwischen Wilhelm und Amelie aufzunehmen, in einem Gastmahl (Abb. 30), zu dem das Schaustechen stattfindet und nach dem das Beilager sämitlicher im Roman geschilderter hochgestellter Persönlichkeiten erfolgt - König und Königin von Frankreich und England sowie Amelie und Wilhelm. Dabei ist wie in den anderen Darstellungen besonderes Gewicht gelegt auf die Konkretisierung der Personen durch die ihnen zugehörigen heraldischen Zeichen.

Die Bilder der Nürnberger Version erzählen zweifellos eine ganz andere Geschichte als diejenigen der anderen Handschriften. Der Roman ist hier zu einem Turnierbuch ausgestaltet, das kraft der Bedeutung der agierenden Helden aus der Antike (in dem vorgebundenen Trojanerkrieg) und dem alten Rittertum (im Wilhelm) Vorbildlichkeitscharakter für das gesamte Turnierwesen gewinnt. Die Banner geben sogar Grund zur Annahme, daß dieses Sammelwerk

$\left.{ }^{66}\right)$ Nürnberg, Germanisches National Museum, Hs. 998 (vgl. Anm. 17).

$\left.{ }^{67}\right)$ Folgende Themen werden dargestellt: fol. 205v/206: Treffen der Heere, Kampf zwischen Wilhelm und Jofrit; 207: Jofrits Flucht, Tod von Wilhelms Vater; 209: Treueeid des Volkes vor dem neugeborenen Kind, Gang Ilyens zum Begräbnis in Begleitung des Königs von Frankreich; 222v: Wilhelm verabschiedet sich vom König von England und dessen Gemahlin, daneben Wilhelm und Amelie.

${ }^{68}$ ) Le Livre de Toumois du Roi René, hrsg. von F. Avril, Paris 1986. - Zu den Tumierbüchern im deutschen Bereich s. O. Gamber, Ritterspiel und Turnierrüstung im Spätmittelalter, in: Das ritterliche Turnier im Mittelalter, hrsg. von J. Fi.eckenstein, Göttingen 1986, S. 513-531, bes. S. 513 f. 519 ff. 
vorbildlicher Kämpfe aus fernen Zeiten zur unmittelbaren Identifizierung mit zeitgenössischen Ereignissen dienen könnte. Im ersten Kolbenturnier, an dem Wilhelm teilnimmt (Abb. 28), könnte das Banner mit dem Mainzer Wappen eine Anspielung auf eines der damals berühmten Turniere sein ${ }^{69}$. Dieser völlig veränderte Interpretationsansatz kann, gerade bei einem Objekt, das von seiner äußeren Ausstattung her den übrigen durchaus entspricht, nur bedeuten, daß derselbe Text hier für einen wiederum anderen Rezeptionszusammenhang konzipiert wurde, eine andere Ansprache ausdrücken wollte.

Anhand dieser Analyse lassen sich nun abschließend folgende grundsätzliche Überlegungen zum Verhältnis von Text und Bild im Medium der Illustration anstellen: An allen Beispielen hat sich zeigen lassen, daß die Illustration weder in einem einseitigen Dienstleistungsverhältnis zum Text steht, noch mit diesem austauschbar ist. Im letzten Fall, der Nürnberger Handschrift, zeichnet sich sogar eine Tendenz ab, dem Bild weitgehende Autonomie zu gewähren. Es stellt eine eigene Sinnschicht dar, die vom Text unabhängig sein kann und diesen vor allem als Vehikel benützt. Zugleich aber ist gerade an diesem Beispiel wiederum die besondere Zusammengehörigkeit der beiden Medien ersichtlich geworden. Wären uns von dieser Handschrift nur die Bilder überliefert, so würden wir sie als Turnierbuch bezeichnen. Der Text erst ordnet die Bilder in einen größeren Zusammenhang ein, gibt ihnen die historische Tiefe und bringt damit ihren besonderen Legitimationsanspruch zum Ausdruck. Die strukturelle Angleichung der beiden Medien in einer gemeinsamen Sprache, in der etwa die lineare Zeitlichkeit des Textes auch vom Bild aufgenommen würde, erfolgt auf sehr unterschiedliche Weise. In den Kursivbildern des frühen 15. Jahrhunderts ist sie sicher beabsichtigt, wogegen sowohl der Münchner Wilhelm als auch die Federzeichnungen der Nürnberger Version jeweils ganz eigenen bildsprachlichen Regeln folgen. In keinem einzigen Fall, auch nicht in den einfachen Kursivbildern des frühen 15. Jahrhunderts, setzen die Bilder bloß den Text in eine andere Sprache um, sie gehen immer über diesen hinaus, sind keine Nachschöpfungen, sondern neue kreative Akte, die für eine spezifische Rezeptionsmöglichkeit in einer bestimmten historischen Situation geschaffen wurden.

69) 1411 wird Mainz als Turnierort erwähnt und gilt sogar 1480 als einer der Hauptorte zur Austragung von Turnieren; s. TH. Zorz, Adel, Bürgertum und Turnier in deutschen Städten vom 13. bis 15. Jahrhundert, in: Das ritterliche Turnier im Mittelalter, hrsg. von J. Fl.eckenstein, Göttingen 1986, S. 450-499, bes. 462- 468.

Abbildungsnachweis: Abb. 1-3, 11, 13, 16, 18: Luzern, E. Gaul. - Abb. 4: Berlin, Staatsbibliothek. - Abb. 5, 28- 30: Nürnberg, German. Nationalmuseum. - Abb. 6: Würzburg, Universitätsbibliothek. - Abb. 7: Wien, Österr. Nationalbibliothek. - Abb. 8, 10, 12, 14: München, Staatsbibliothek. - Abb. 9: Heidelberg, Universitätsbibliothek. Abb. 15: Den Haag, Koninklijke Bibliotheek. - Abb. 17: Freiburg i. B., Stadtarchiv. - Abb. 19-26: Stuttgart, Landesbibliothek. - Abb. 27: Wiesbaden, Landesbibliothek.

Allen Bibliotheken, die mir großzügig das Material zur Verfügung gestellt haben, sei herzlich gedankt. 


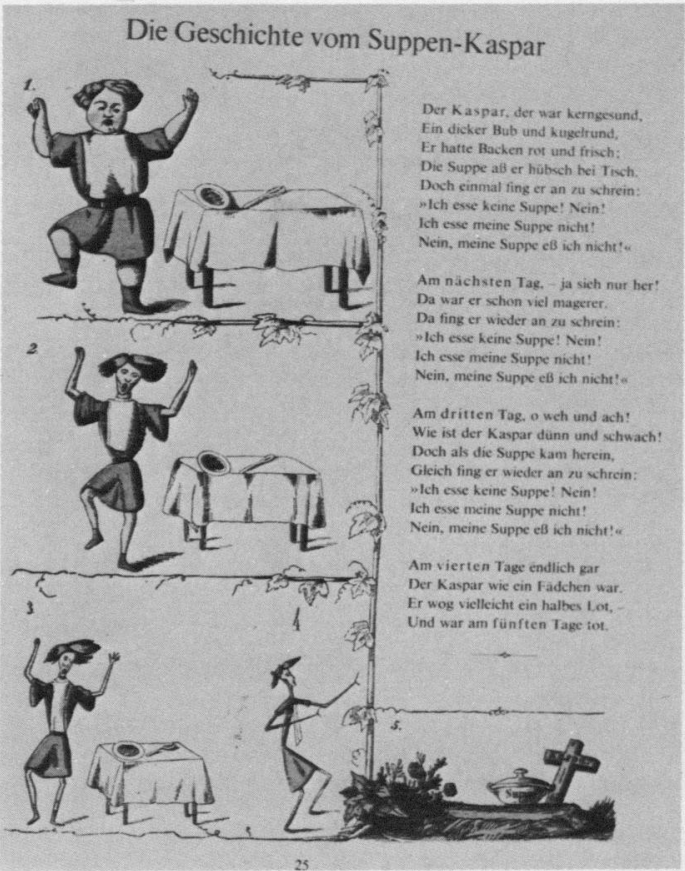

1. Suppen-Kaspar, Dr. Heinrich Hoffmanns Struwwelpeter, 100. Auflage, 1876

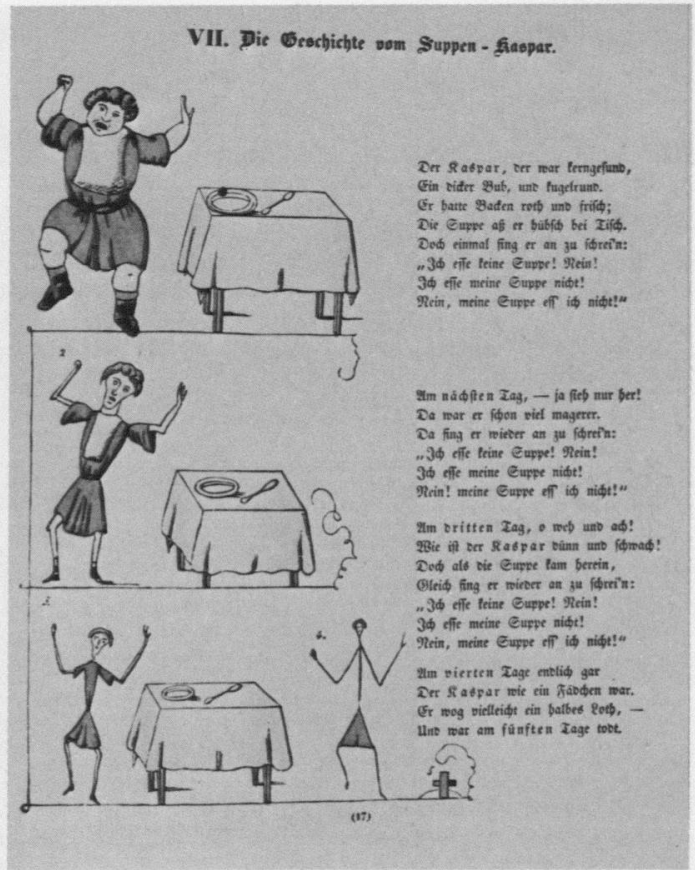

2. Suppen-Kaspar, Dr. Heinrich Hoffmanns Struwwelpeter, 24. Auflage, 1847

3. Suppen-Kaspar, Dr. Heinrich Hoffmanns Struwwelpeter, 1981

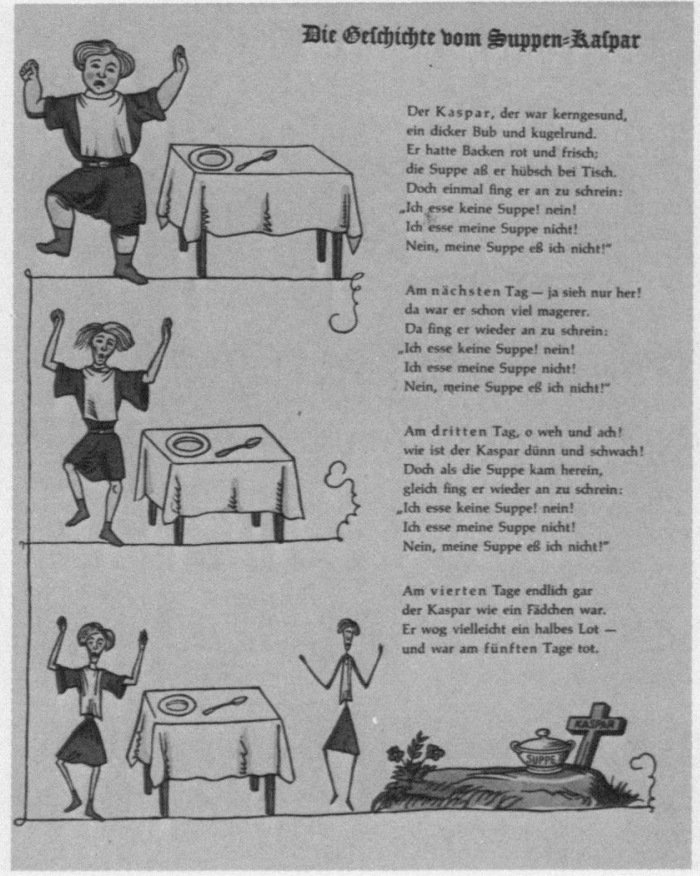



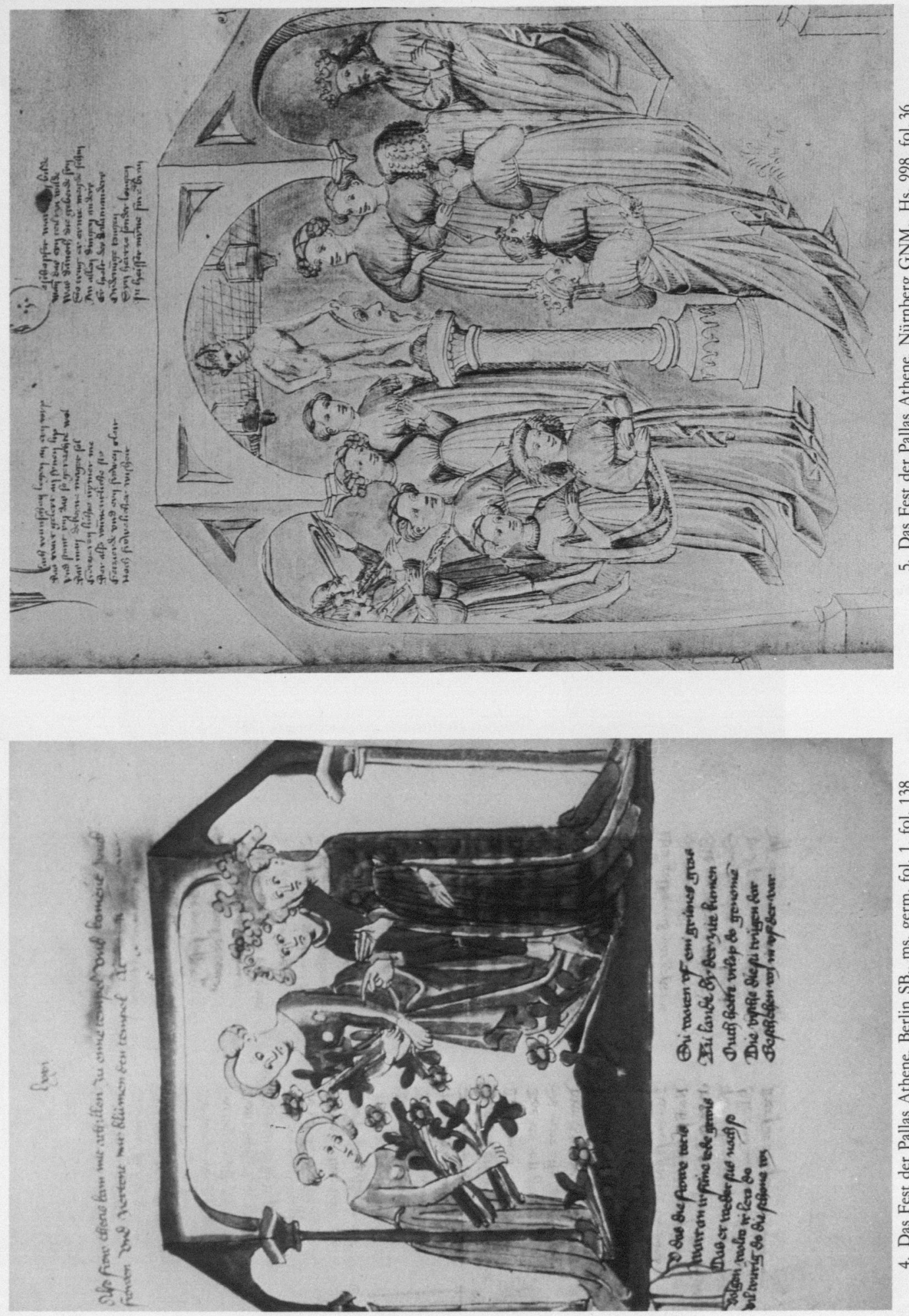

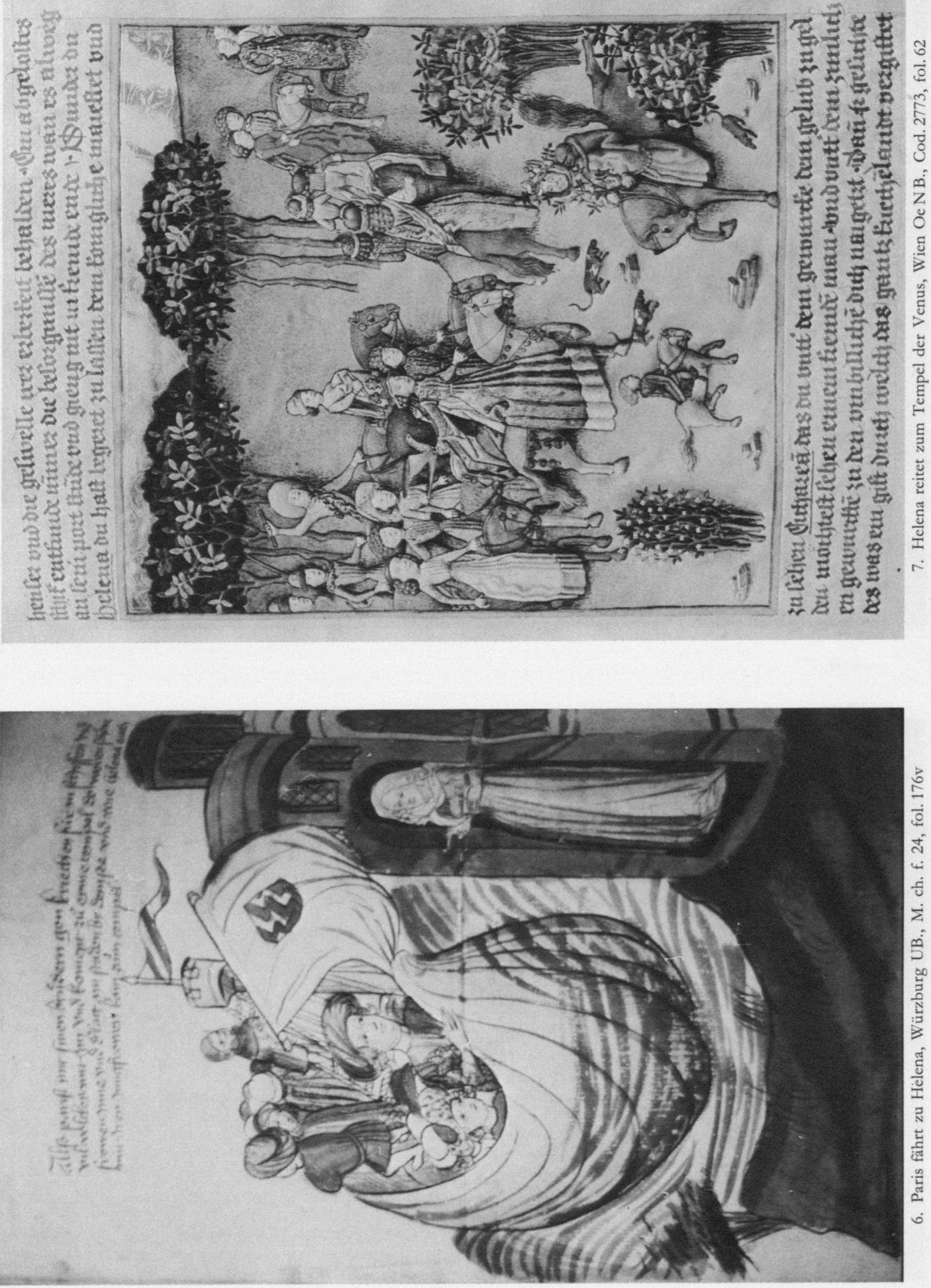



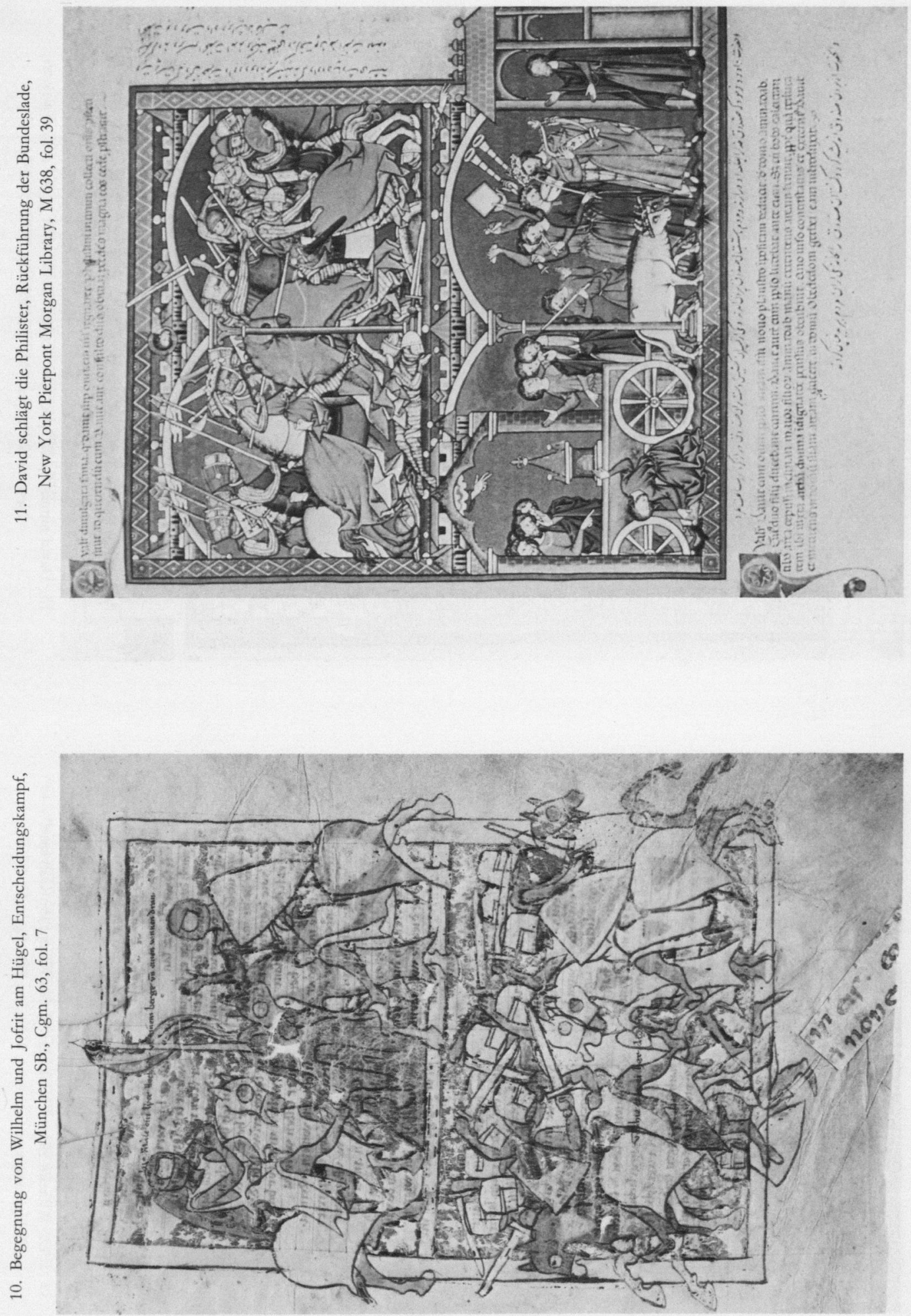

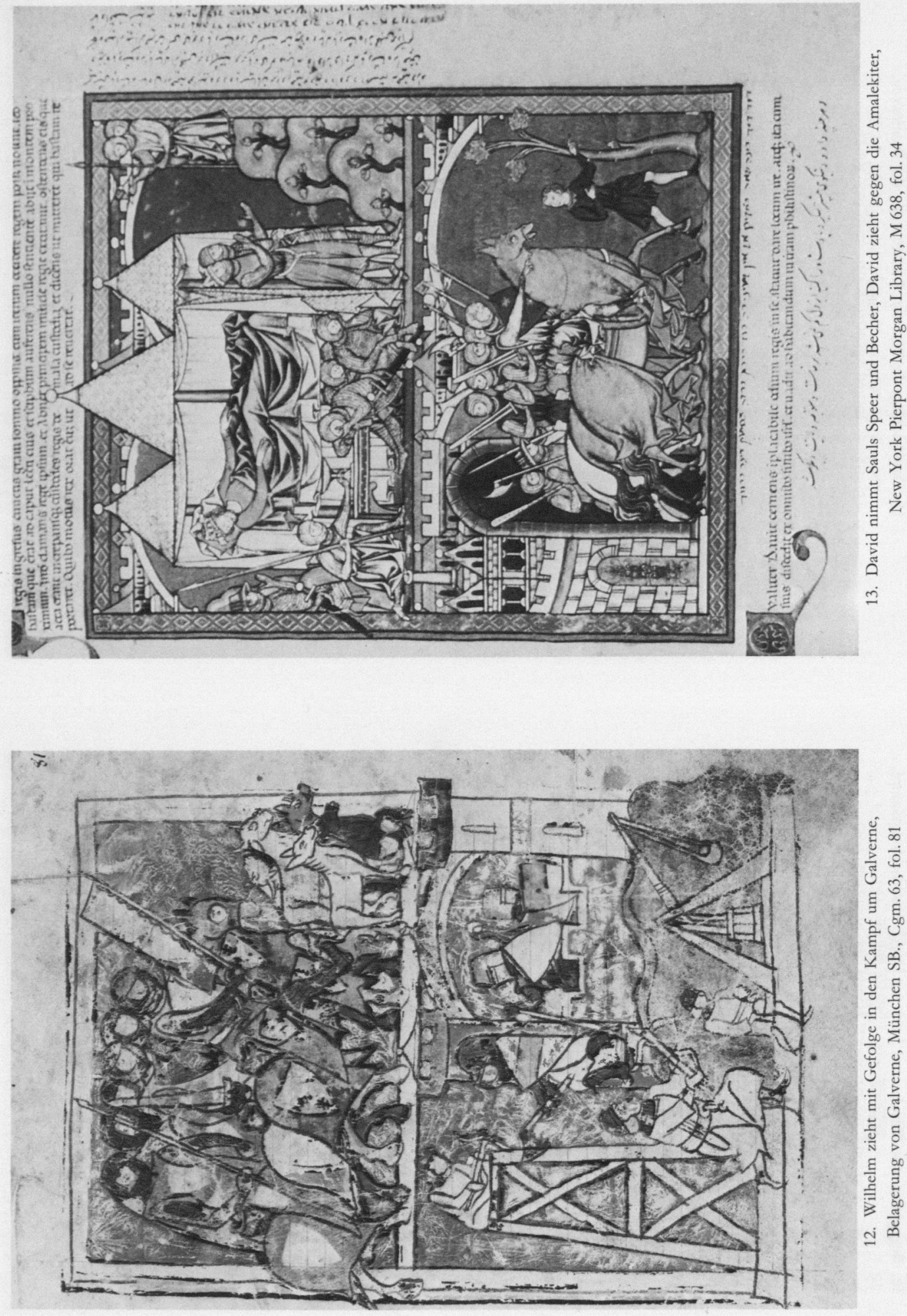

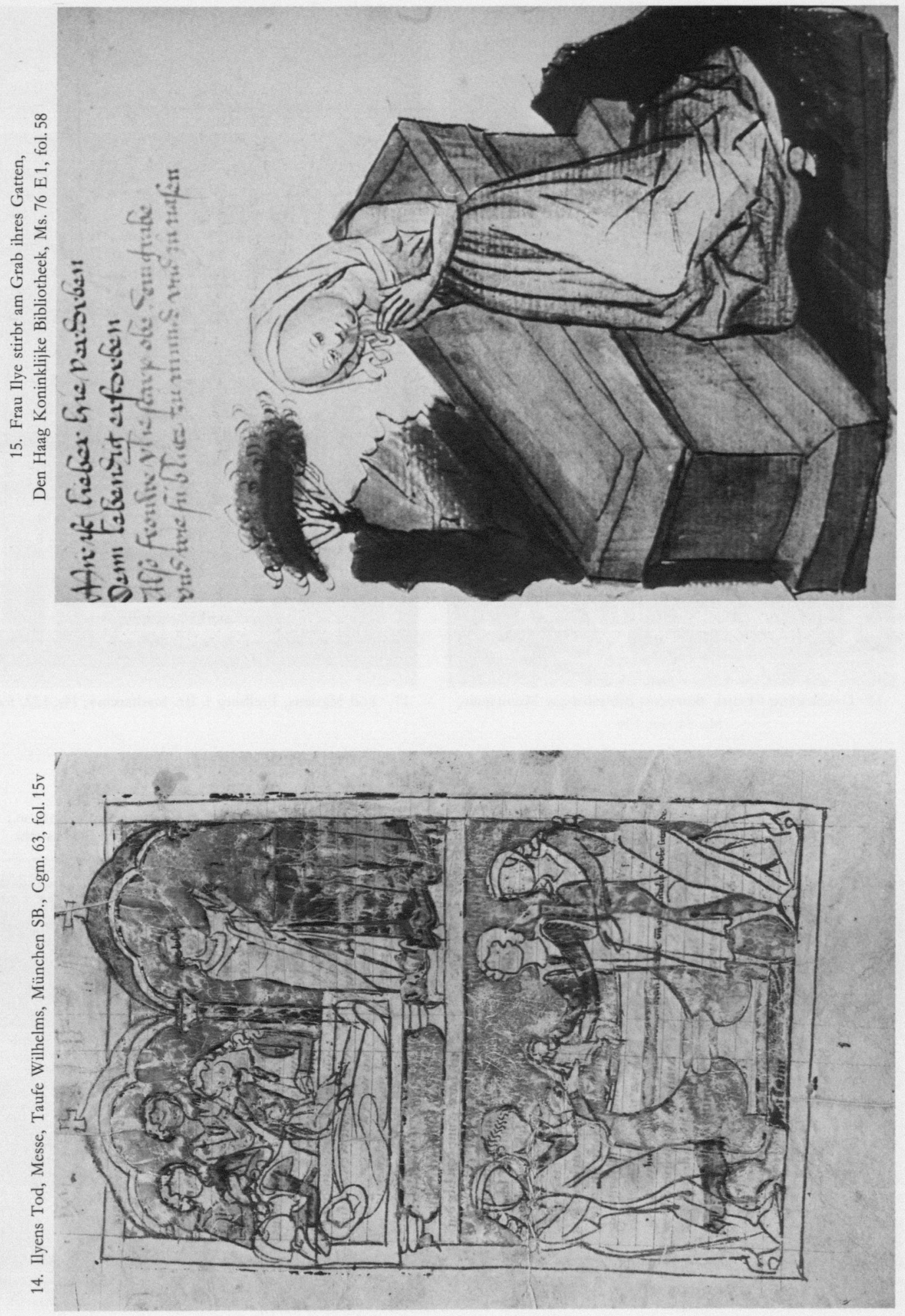


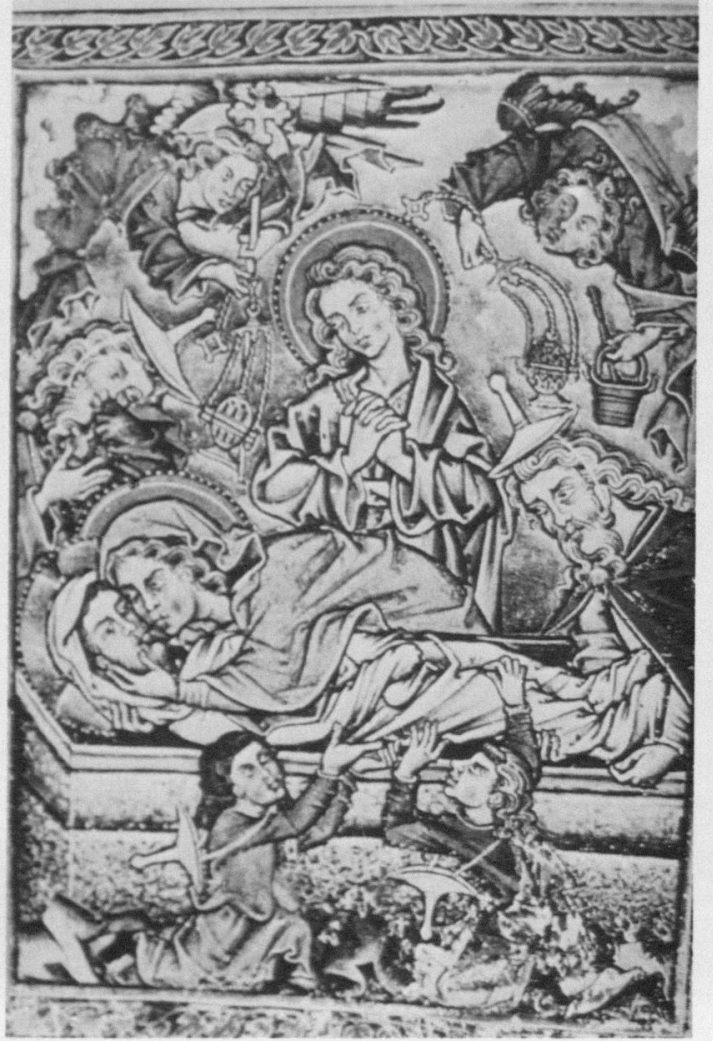

16. Grablegung Christi, Besançon Bibliothèque Municipale,



17. Tod Mariens, Freiburg i. Br. Stadtarchiv, Hs. 122, fol. 31

Ms. 54 , fol. $17 \mathrm{v}$

18. Wilhelm und Amelie beim Schach, Wilhelm und Amelie vor der Kapelle, Wilhelms Minnekrankheit, Amelie besucht den Kranken, Abschied. Detail aus dem Wilhem von Orlens-Teppich, Frankfurt Kunstgewerbemuseum

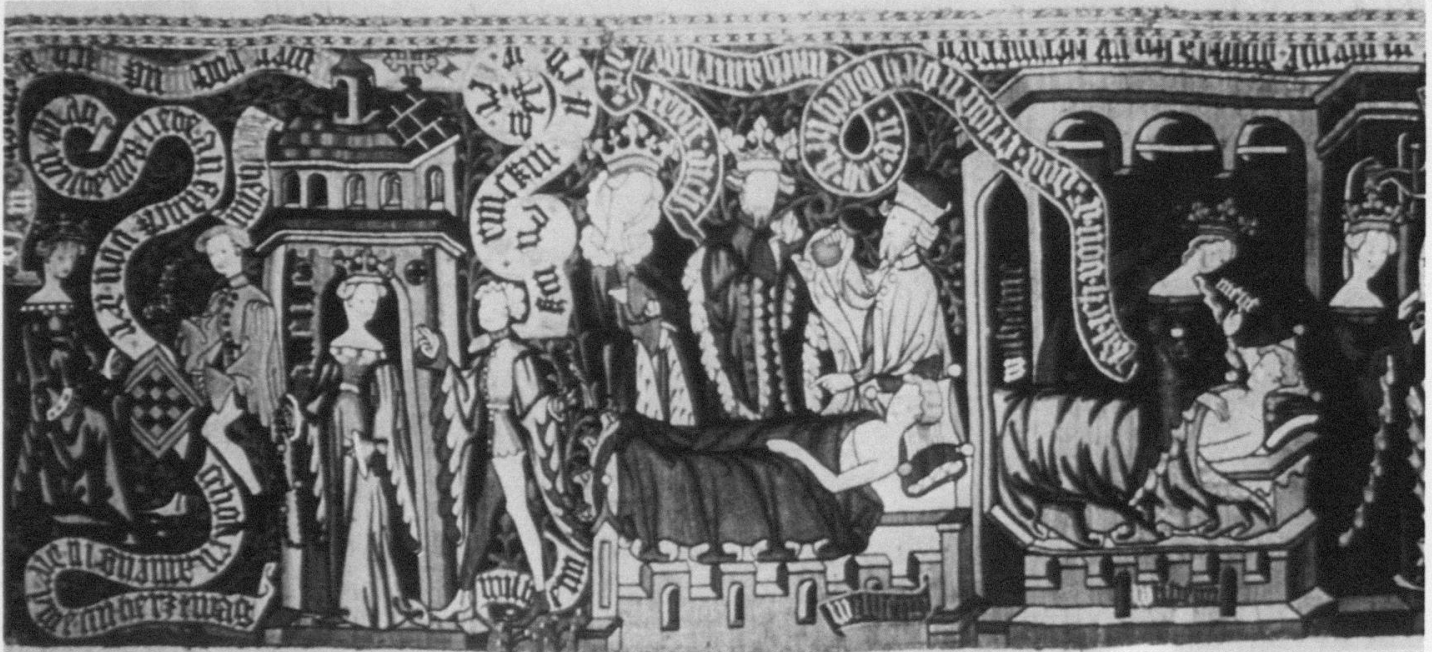




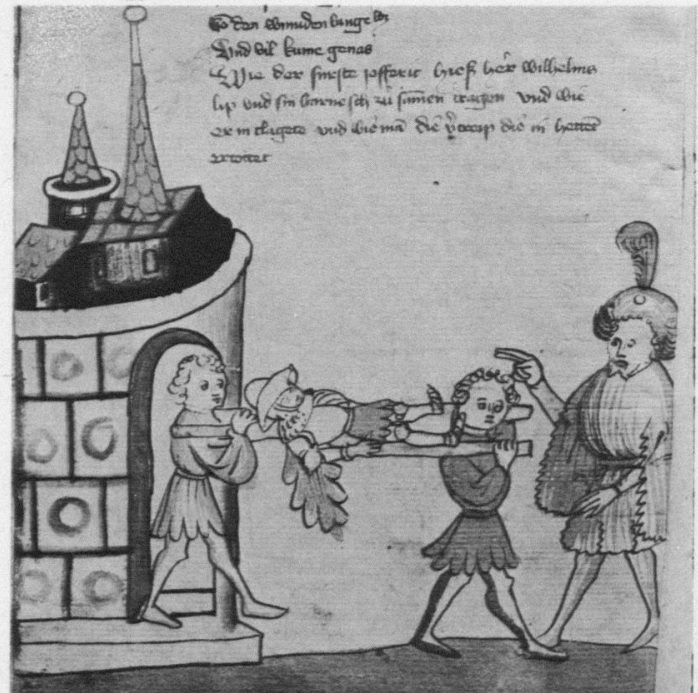

19. Der tote Wilhelm auf der Bahre vor Jofrit, Stuttgart LB., H B XIII 2, fol. 32v

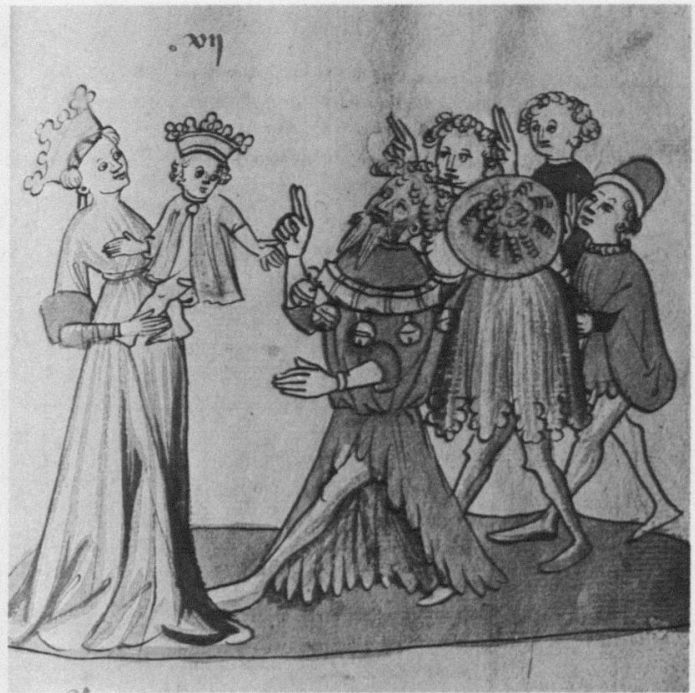

20. Die Untertanen schwören Wilhelm den Treueeid, Stuttgart LB., H B XIII 2, fol. 37
21. Die Brabanter anerkennen Wilhelm als Jofrits Nachfolger, Stuttgart LB., H B XIII 2, fol. 56

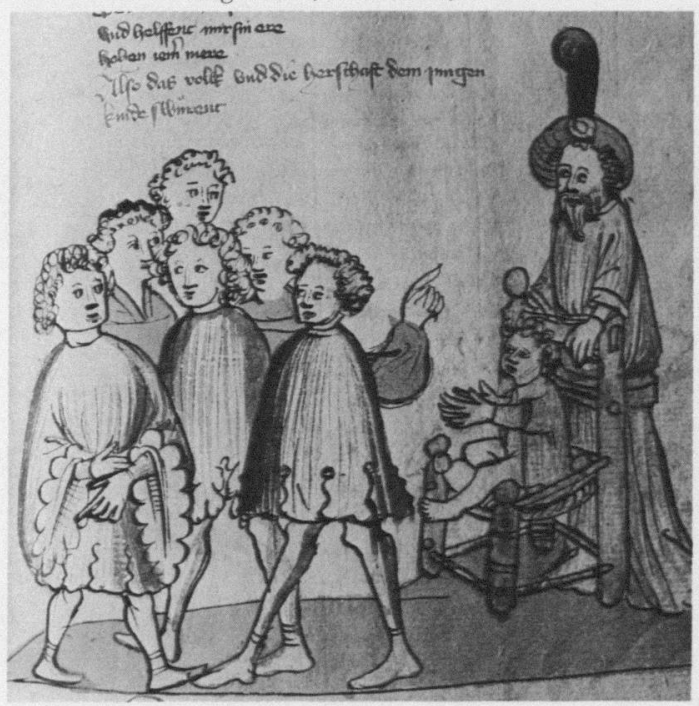

22. Wilhelm erhält vom Kaiser das Fahnlehen, Stuttgart LB., H B XIII 2, fol. 64

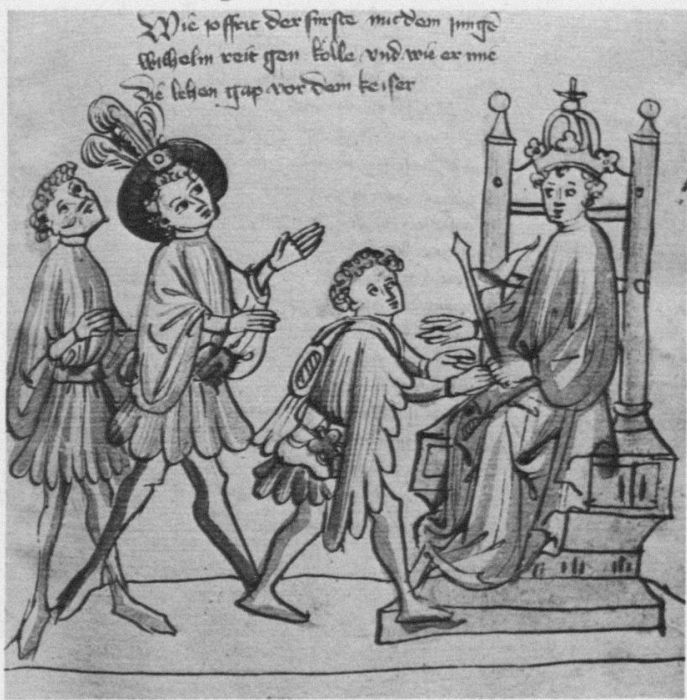






23. Wilhelm erhält vom Kaiser den Lehensbrief, Stuttgart LB., H B XIII 2, fol. $65 \mathrm{v}$

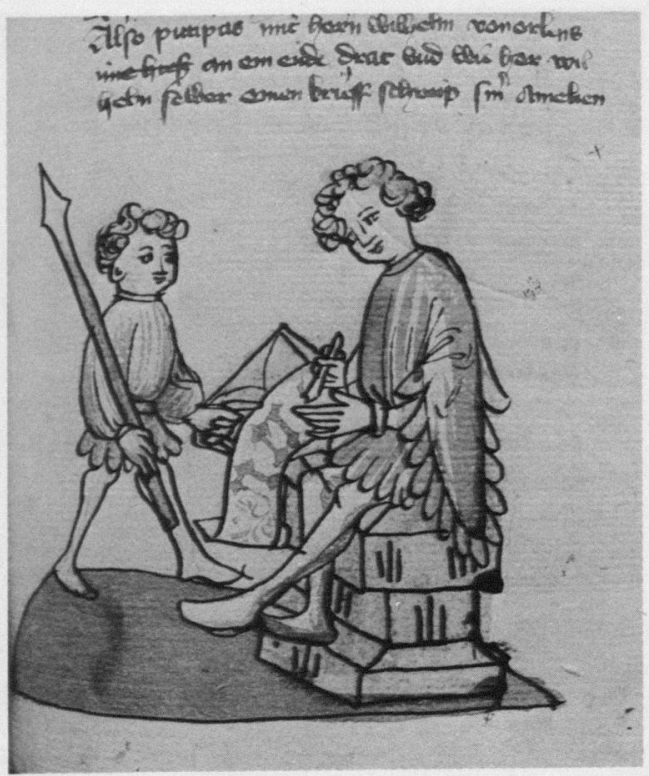

24. Wilhelm schreibt Amelie einen Brief, daneben Pittipasz, Stuttgart LB., H B XIII 2, fol. 159v
25. Wilhelm beruft den Rat ein zur Entscheidung gegen Jofrit, Stuttgart LB., H B XIII 2, fol. 21

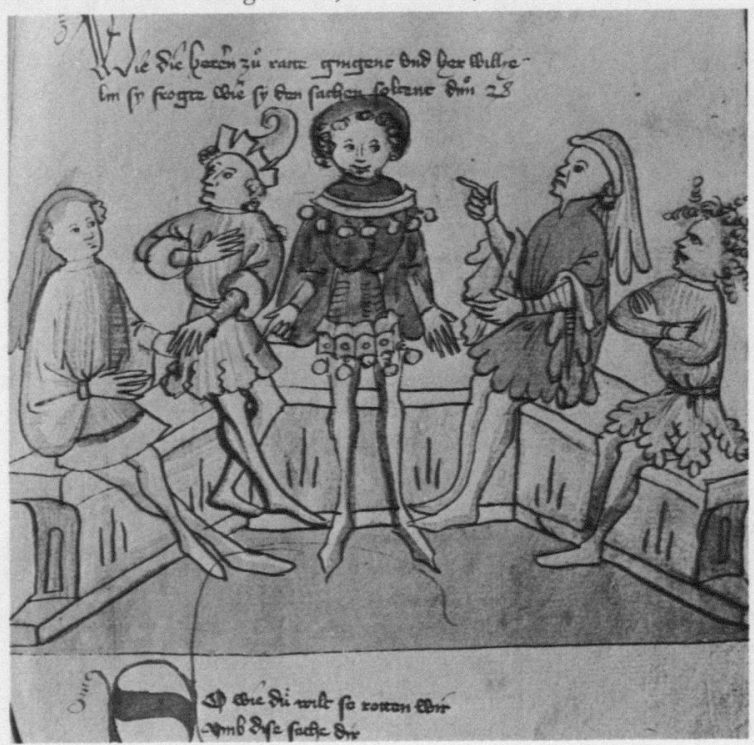

26. Wilhelm legt seinen Eid vor Amelie ab, Stuttgart LB., H B XIII 2, fol. 96



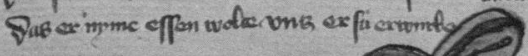

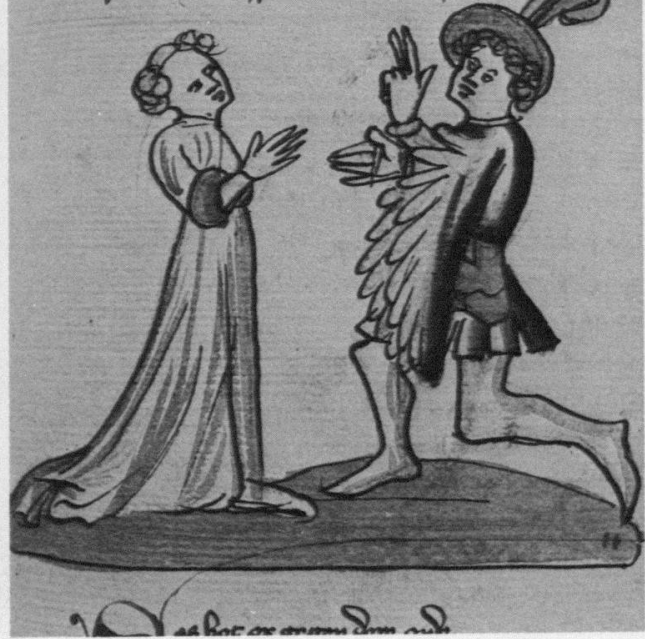


27. Moses läßt das Buch Jesajas als Rechtsbeweis vor Salomo, den Richter bringen, Wiesbaden LB., $\mathrm{Hs} \mathrm{Nr}$.66, fol. $48 v / 49$
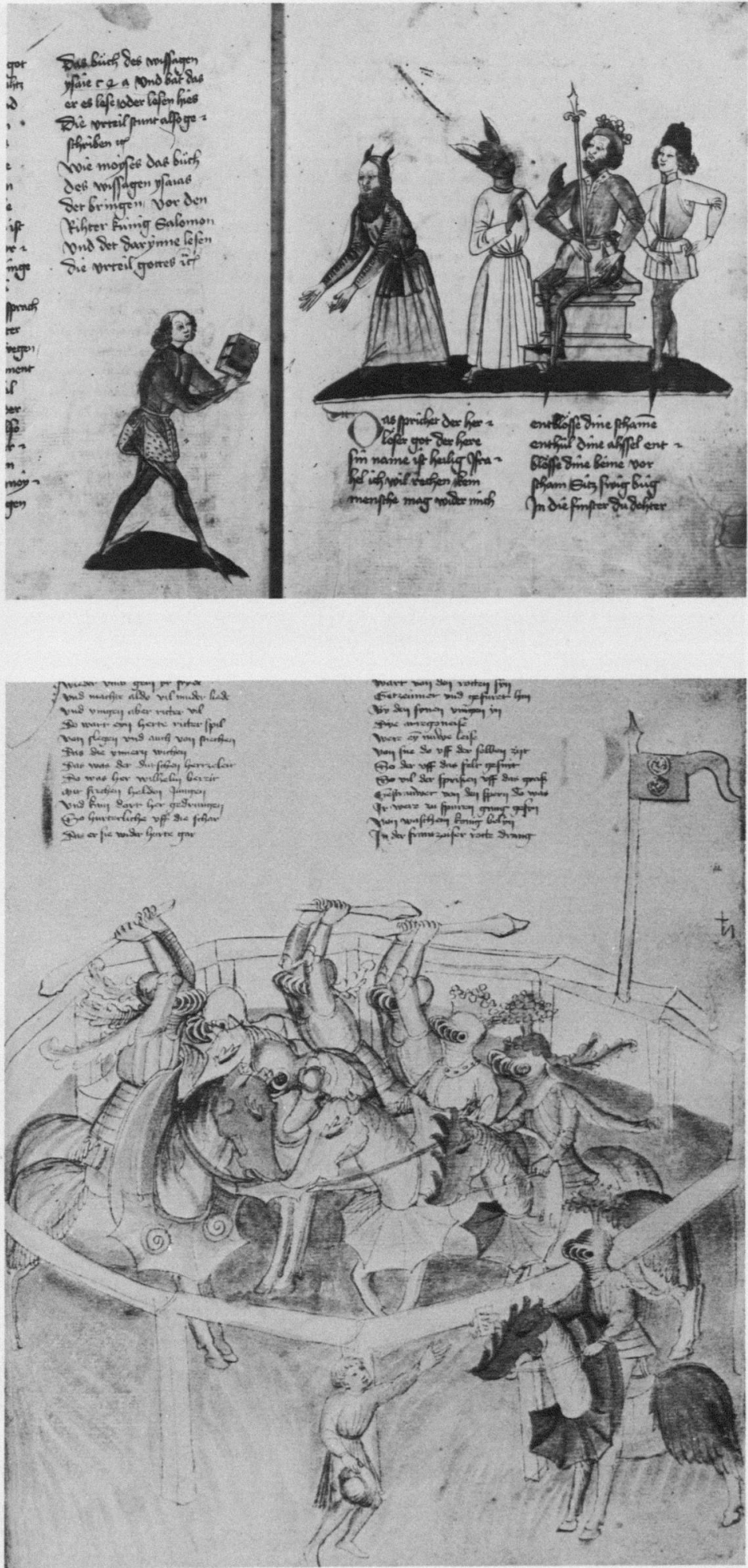

28. Wilhelms Bewährung im Turnier, Wilhelm übergibt Pittipasz den Brief, Nürnberg GNM., Hs. 998, fol. 227 

